Supporting Information for:

\title{
Non-Directed C-H Activation of Arenes with Cp*Ir(III) Acetate Complexes: An Experimental and Computational Study
}

Daniel A. Frasco ${ }^{\dagger}$, Sriparna Mukherjee ${ }^{\dagger}$, Roger D. Sommer ${ }^{\dagger}$, Cody M. Perry ${ }^{\dagger}$, Nikola S. Lambic $^{\dagger}$, Khalil A. Abboud ${ }^{\ddagger}$, Elena Jakubikova ${ }^{\dagger *}$ and Elon A. Ison ${ }^{\dagger *}$

'Department of Chemistry, North Carolina State University, 2620 Yarbrough Drive, Raleigh, NC 276958204 and Center for Catalysis, University of Florida, P.O. Box 117200, Gainesville, Florida 32611, United States 


\section{Table of Contents}

KINETIC DATA

S3-S6

ADDITIONAL SPECTROSCOPIC DATA FOR 8 AND 9

S7-S10

COMPUTATIONAL DATA

S11-S15

X-RAY DATA

S16-S60 


\section{ADDITIONAL KINETIC DATA}

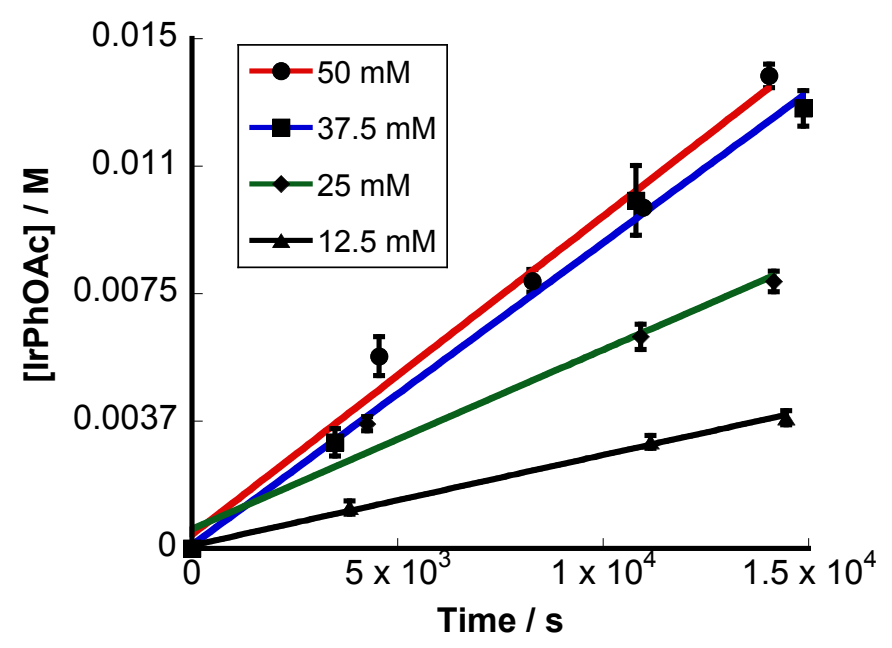

Figure S1. Plot of concentration of $\mathbf{6}$ vs time using different concentrations of $\mathbf{1}$. Initial rates were determined with 1 at 12.5, 25, 37.5 and $50 \mathrm{mM}$ with 5 equiv $\mathrm{Et}_{3} \mathrm{~N}$ with respect to 1 and $1 \mathrm{~mL}$ benzene at $60{ }^{\circ} \mathrm{C}$. Results were obtained in at least triplicate by ${ }^{1} \mathrm{H}$ NMR analysis of the crude reaction mixture using mesitylene as an internal standard.

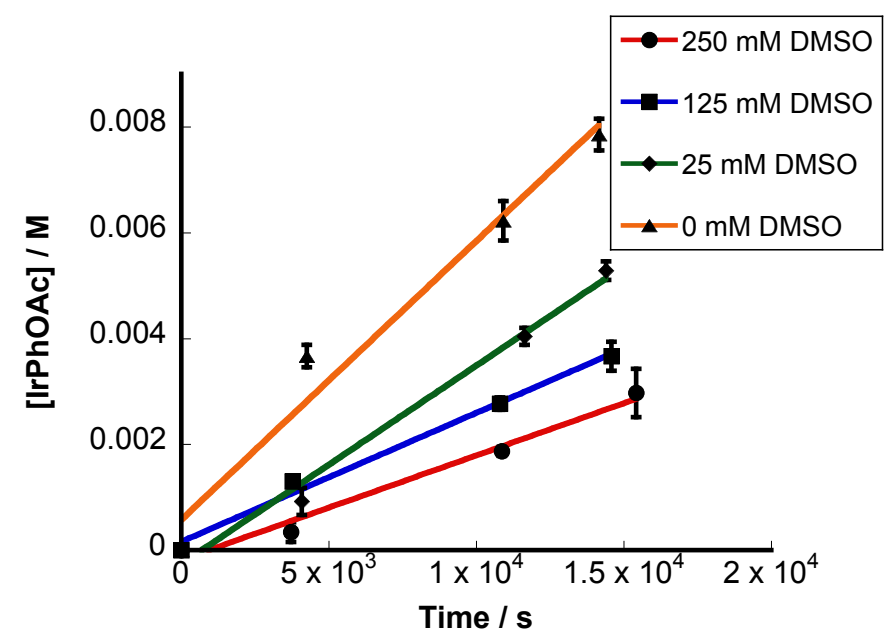

Figure S2. Plot of concentration of $\mathbf{6}$ vs time using different concentrations of DMSO. Initial rates were determined with DMSO at 25,125 , and $250 \mathrm{mM}, 25 \mathrm{mM}, \mathbf{1}$, with 125 $\mathrm{mM} \mathrm{Et}{ }_{3} \mathrm{~N}$ and $1 \mathrm{~mL}$ benzene at $60{ }^{\circ} \mathrm{C}$. Results were obtained in at least triplicate by ${ }^{1} \mathrm{H}$ NMR analysis of the crude reaction mixture using mesitylene as an internal standard. 


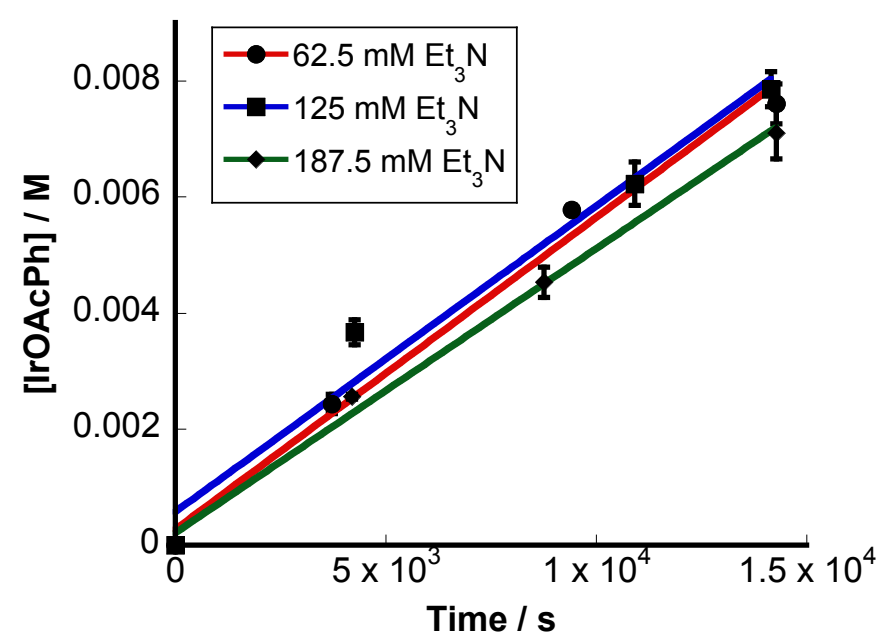

Figure S3. Plot of concentration of 6 vs time using different concentrations of $\mathrm{Et}_{3} \mathrm{~N}$. Initial rates were determined with $\mathrm{Et}_{3} \mathrm{~N}$ at $62.5,125$, and $187.5 \mathrm{mM}, 25 \mathrm{mM}, 1$, and $1 \mathrm{~mL}$ benzene at $60{ }^{\circ} \mathrm{C}$. Results were obtained in at least triplicate by ${ }^{1} \mathrm{H}$ NMR analysis of the crude reaction mixture using mesitylene as an internal standard.

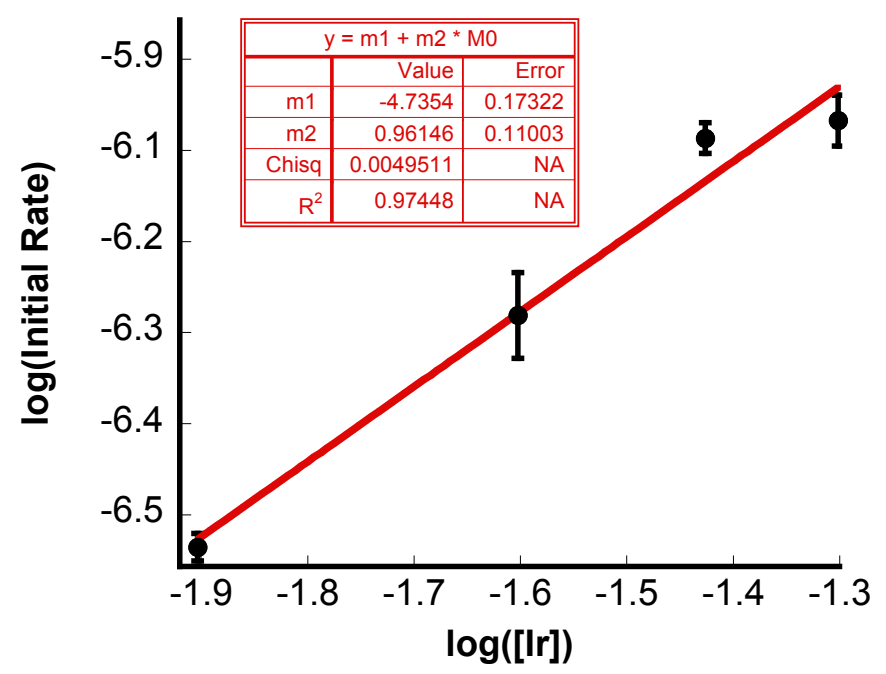

Figure S4. Plot of $\log ($ initial rate) vs $\log ([\operatorname{Ir}])$. Initial rates were determined with $\mathbf{1}$ at $12.5,25,37.5$ and $50 \mathrm{mM}$ with 5 equiv $\mathrm{Et}_{3} \mathrm{~N}$ with respect to 1 and $1 \mathrm{~mL}$ benzene at 60 ${ }^{\circ} \mathrm{C}$. Results were obtained in at least triplicate by ${ }^{1} \mathrm{H}$ NMR analysis of the crude reaction mixture using mesitylene as an internal standard. 


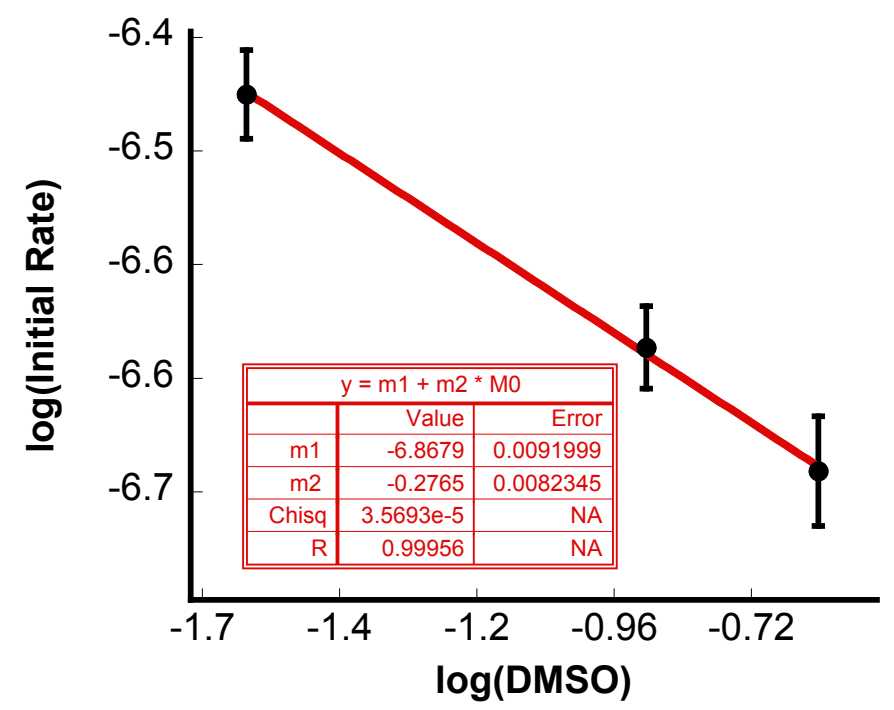

Figure S5. Plot of $\log ($ initial rate) vs $\log (\mathrm{DMSO})$. Initial rates were determined with DMSO at 25,125 , and $250 \mathrm{mM}, 25 \mathrm{mM}, 1,125 \mathrm{mM} \mathrm{Et}_{3} \mathrm{~N}$ and $1 \mathrm{~mL}$ benzene at $60{ }^{\circ} \mathrm{C}$. Results were obtained in at least triplicate by ${ }^{1} \mathrm{H}$ NMR analysis of the crude reaction mixture using mesitylene as an internal standard.

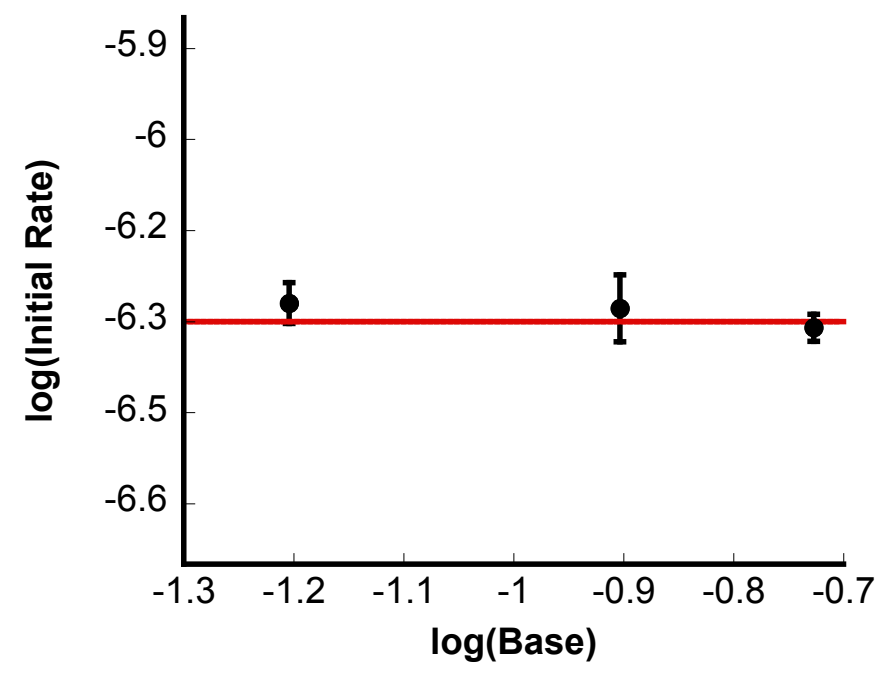

Figure S6. Plot of $\log$ (initial rate) vs $\log \left(\right.$ base). Initial rates were determined with $\mathrm{Et}_{3} \mathrm{~N}$ at $62.5,125$, and $187.5 \mathrm{mM}, 25 \mathrm{mM}, 1$ and $1 \mathrm{~mL}$ benzene at $60^{\circ} \mathrm{C}$. Results were obtained in at least triplicate by ${ }^{1} \mathrm{H}$ NMR analysis of the crude reaction mixture using mesitylene as an internal standard. 


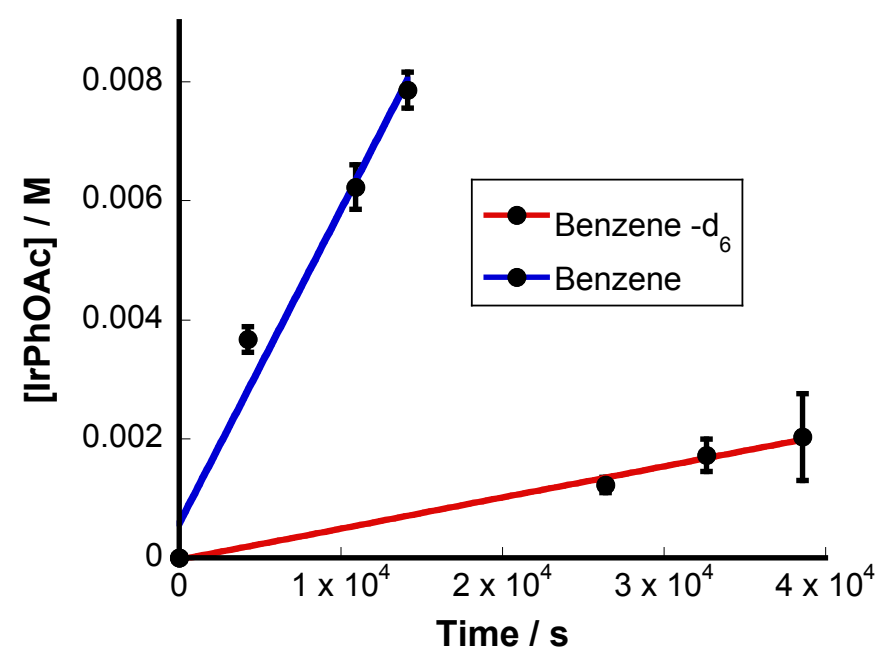

Figure S7. Plot of concentration of 6 vs time using benzene or benzene- $d_{6}$. Initial rates were determined with $25 \mathrm{mM}, 1,125 \mathrm{mM} \mathrm{Et}_{3} \mathrm{~N}$ and $1 \mathrm{~mL}$ benzene or benzene- $\mathrm{d}_{6}$ at 60 ${ }^{\circ} \mathrm{C}$. Results were obtained in at least triplicate by ${ }^{1} \mathrm{H}$ NMR analysis of the crude reaction mixture using mesitylene as an internal standard. A KIE of $k_{\mathrm{H}} / k_{\mathrm{D}}=10.0$ (0.6) was obtained.

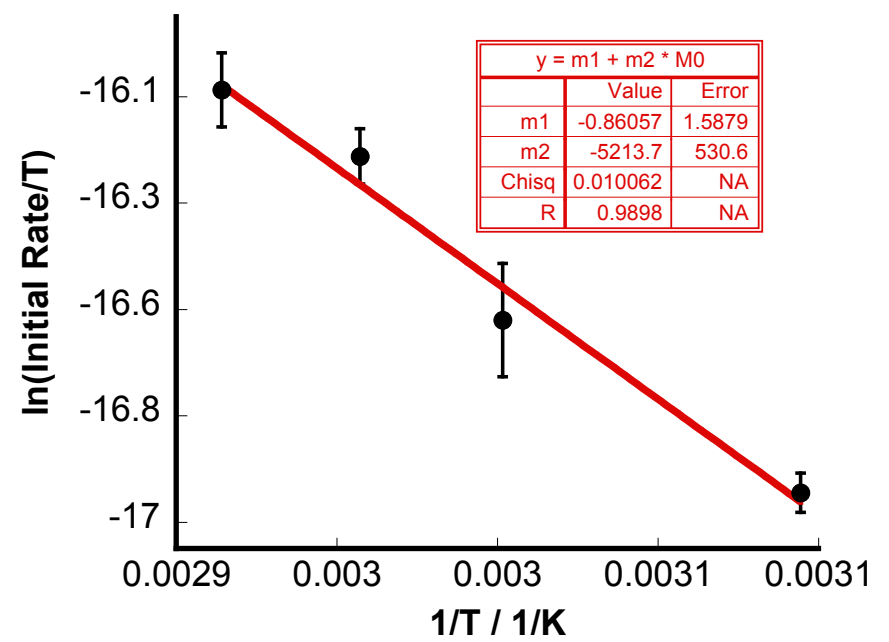

Figure S8. Eyring plot of $\ln ($ Initial Rate/T) vs $1 / \mathrm{T}$. Initial rates were determined with 25 $\mathrm{mM} \mathrm{1,} 125 \mathrm{mM} \mathrm{Et}_{3} \mathrm{~N}$ and $1 \mathrm{~mL}$ benzene or at 50, 60, 65 and $70{ }^{\circ} \mathrm{C}$. Results were obtained in at least triplicate by ${ }^{1} \mathrm{H}$ NMR analysis of the crude reaction mixture using mesitylene as an internal standard. At $60{ }^{\circ} \mathrm{C}$ the reaction was found to have the following parameters: $\Delta \mathrm{H}^{*}=10.3(1.0) \mathrm{kcal} / \mathrm{mol}, \Delta \mathrm{S}^{\star}=-0.049(0.003) \mathrm{kcal} / \mathrm{mol} \mathrm{K}$ and $\Delta \mathrm{G}^{\star}=26.6$ (2.0) $\mathrm{kcal} / \mathrm{mol}$. 


\section{ADDITIONAL SPECTROSCOPIC DATA FOR 8 AND 9}

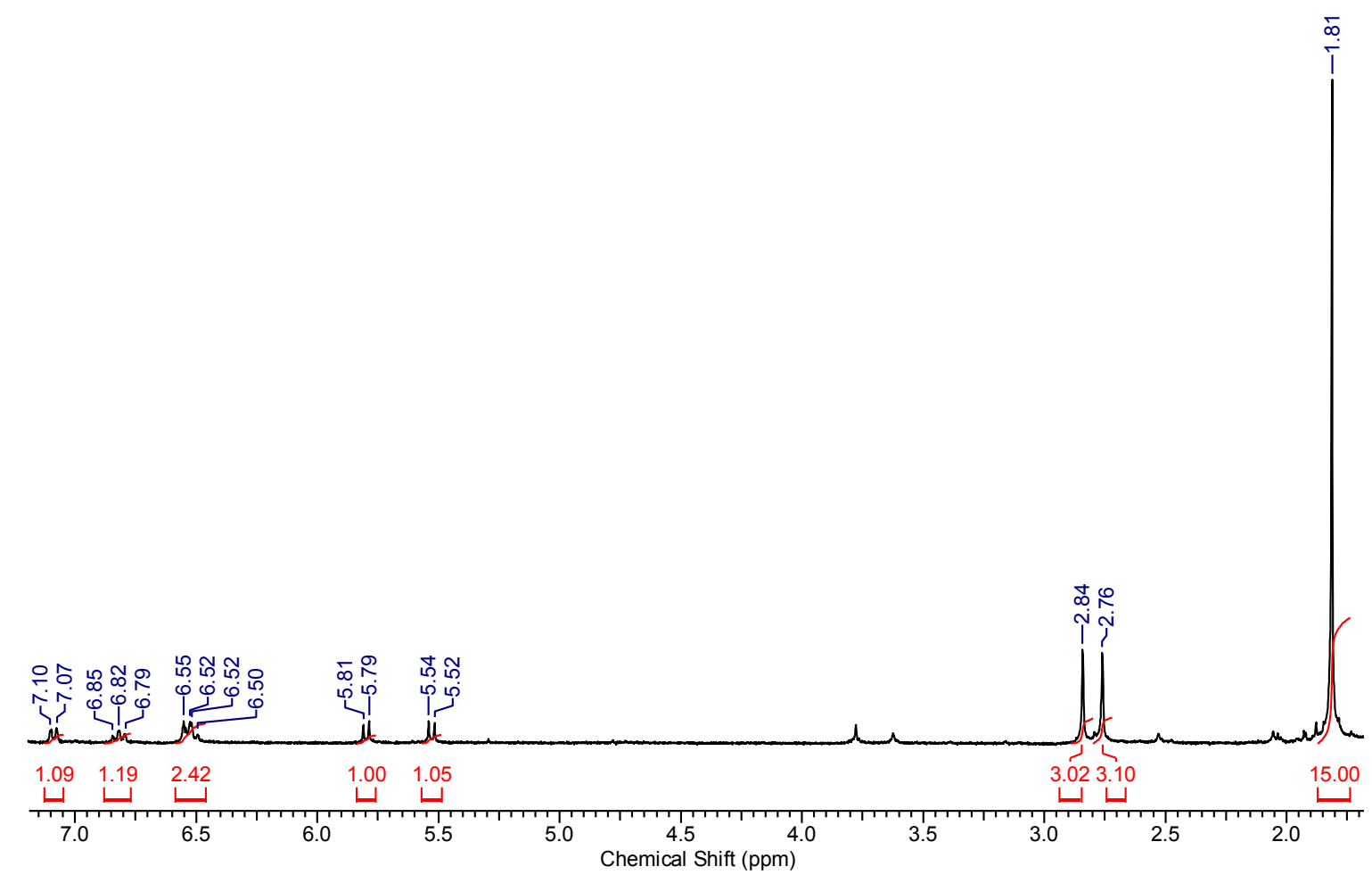

Figure S9. ${ }^{1} \mathrm{H}$ NMR spectrum for 8 in $\mathrm{CDCl}_{3}$ 


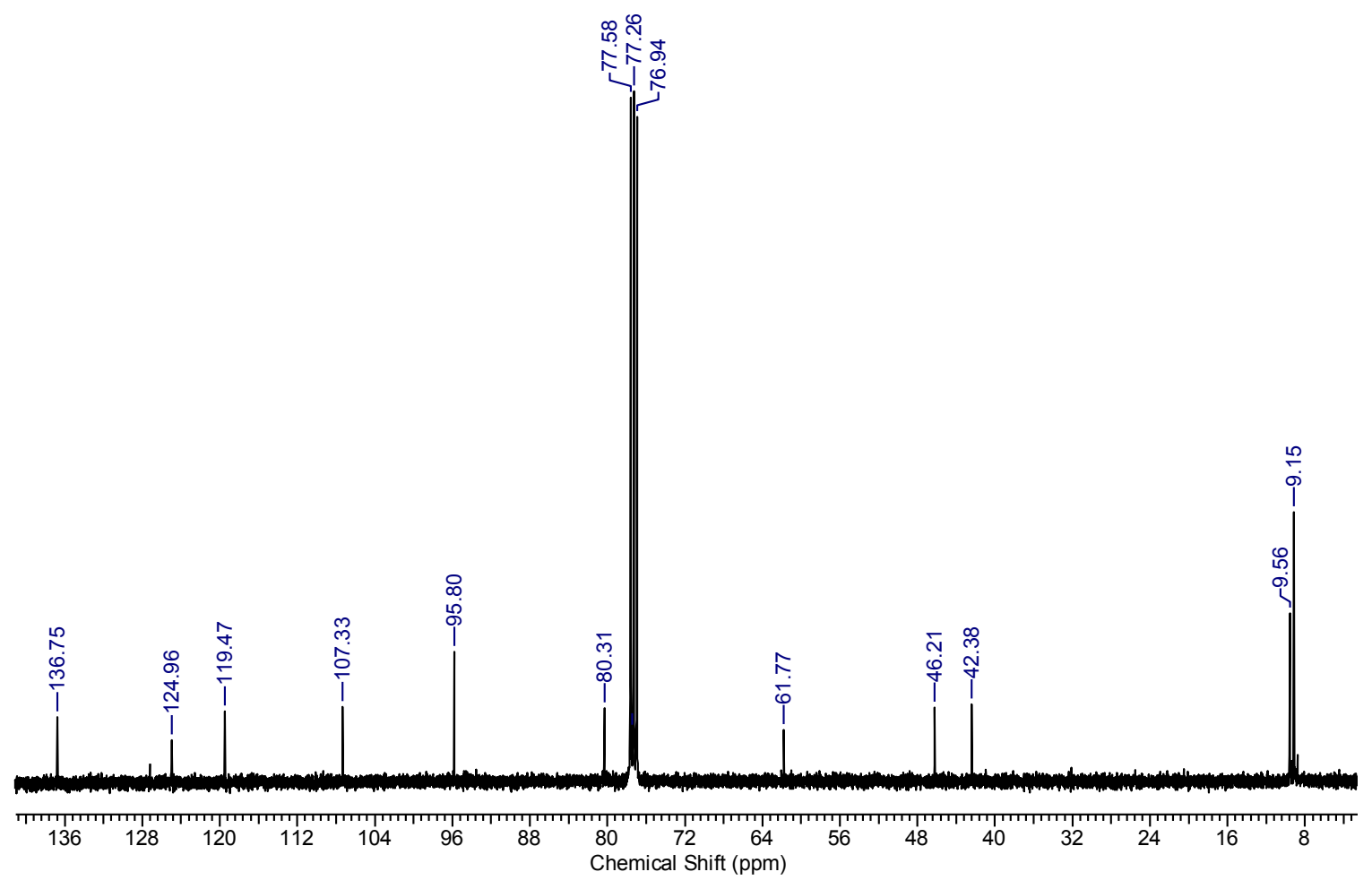

Figure S10. ${ }^{13} \mathrm{C}$ NMR spectrum for 8 in $\mathrm{CDCl}_{3}$ 


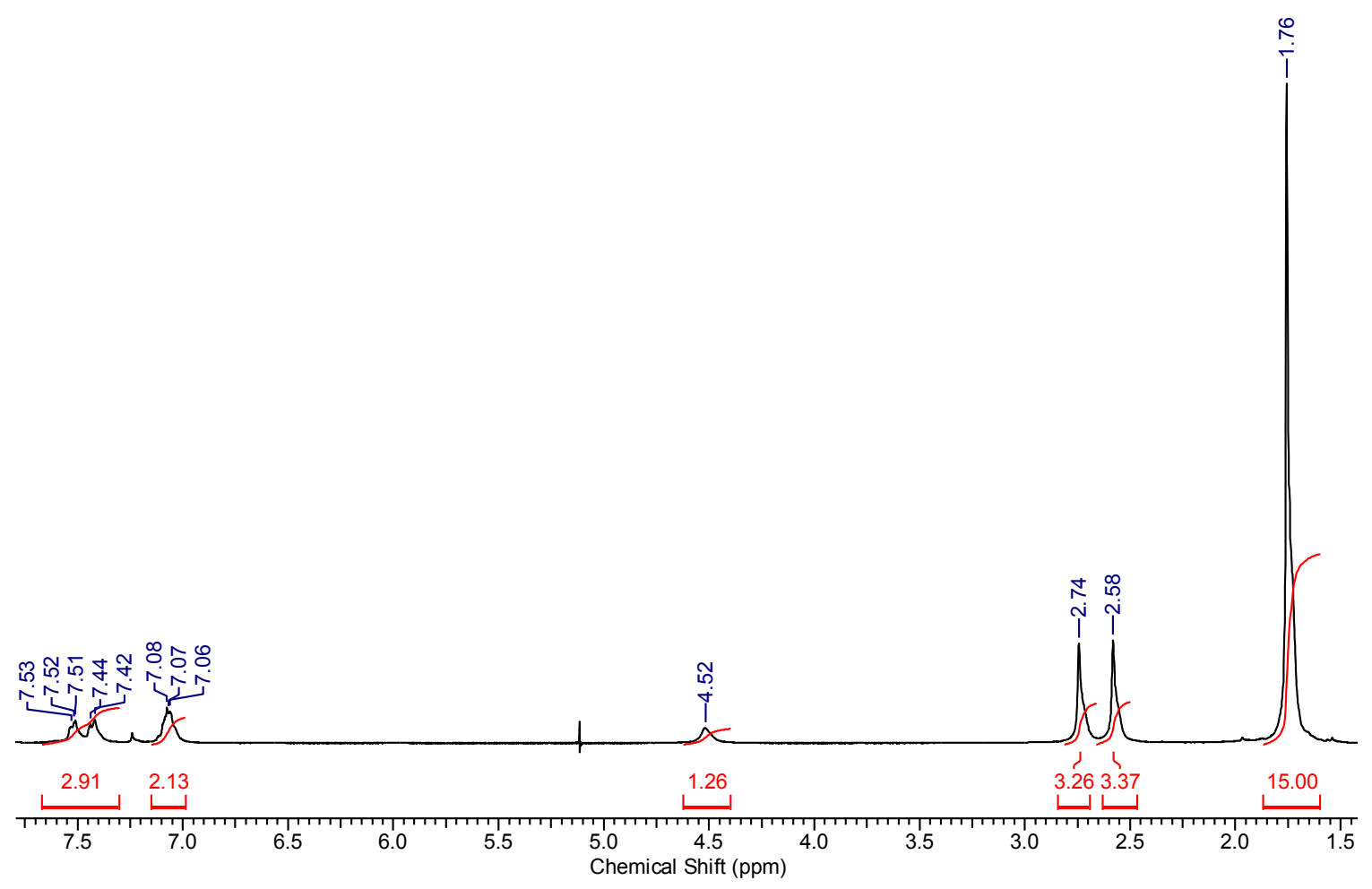

Figure S11. ${ }^{1} \mathrm{H}$ NMR spectrum for 9 in $\mathrm{CDCl}_{3}$ 


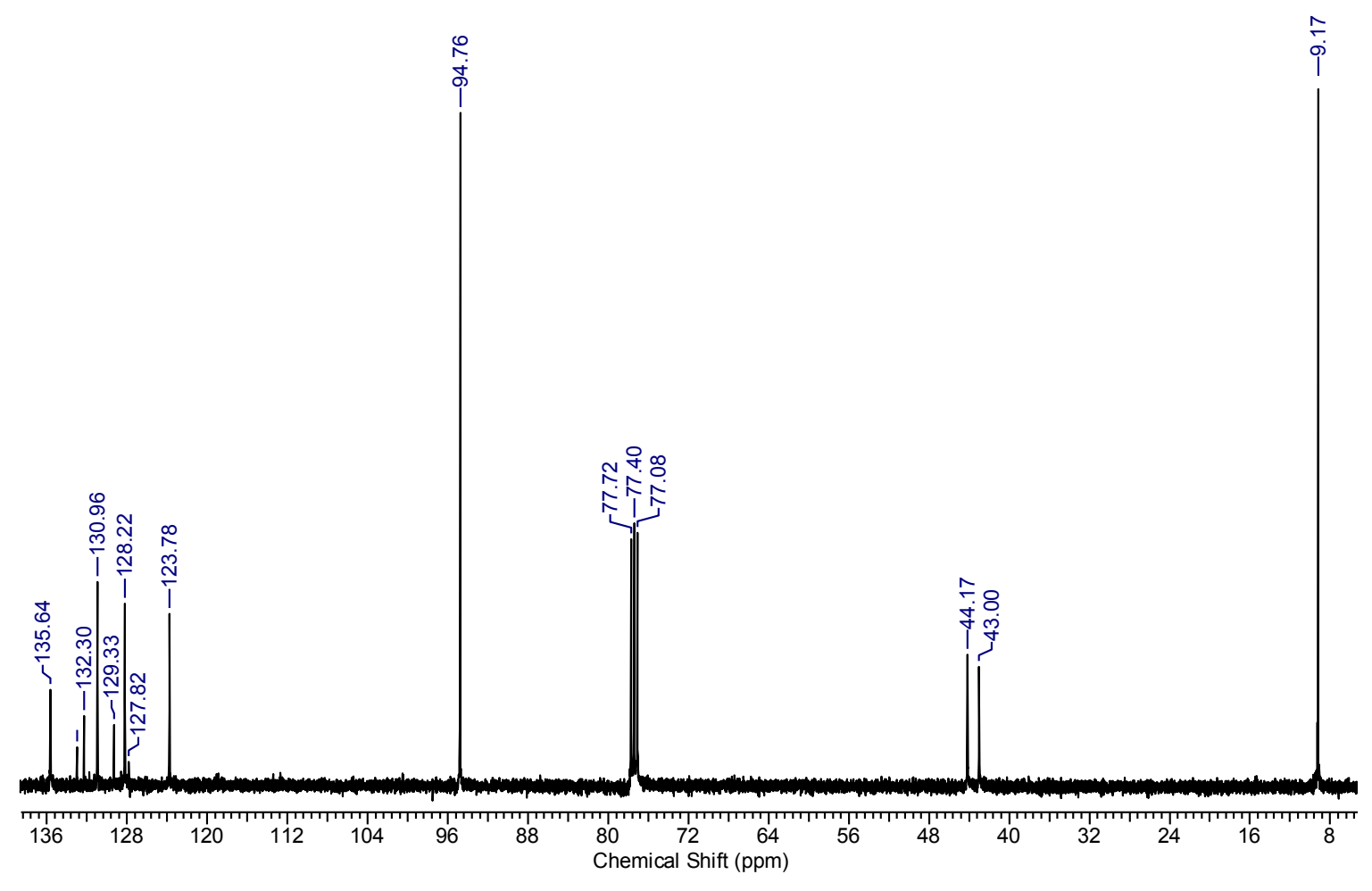

Figure S12. ${ }^{13} \mathrm{C}$ NMR spectrum of 9 in $\mathrm{CDCl}_{3}$ 


\section{COMPUTATIONAL DATA}

\section{Full Gaussian09 Citation}

Gaussian 09, Revision D.01, Frisch, M. J.; Trucks, G. W.; Schlegel, H. B.; Scuseria, G. E.; Robb, M. A.; Cheeseman, J. R.; Scalmani, G.; Barone, V.; Mennucci, B.; Petersson, G. A.; Nakatsuji, H.; Caricato, M.; Li, X.; Hratchian, H. P.; Izmaylov, A. F.; Bloino, J.; Zheng, G.; Sonnenberg, J. L.; Hada, M.; Ehara, M.; Toyota, K.; Fukuda, R.; Hasegawa, J.; Ishida, M.; Nakajima, T.; Honda, Y.; Kitao, O.; Nakai, H.; Vreven, T.; Montgomery, J. A., Jr.; Peralta, J. E.; Ogliaro, F.; Bearpark, M.; Heyd, J. J.; Brothers, E.; Kudin, K. N.; Staroverov, V. N.; Kobayashi, R.; Normand, J.; Raghavachari, K.; Rendell, A.; Burant, J. C.; Iyengar, S. S.; Tomasi, J.; Cossi, M.; Rega, N.; Millam, M. J.; Klene, M.; Knox, J. E.; Cross, J. B.; Bakken, V.; Adamo, C.; Jaramillo, J.; Gomperts, R.; Stratmann, R. E.; Yazyev, O.; Austin, A. J.; Cammi, R.; Pomelli, C.; Ochterski, J. W.; Martin, R. L.; Morokuma, K.; Zakrzewski, V. G.; Voth, G. A.; Salvador, P.; Dannenberg, J. J.; Dapprich, S.; Daniels, A. D.; Farkas, Ö.; Foresman, J. B.; Ortiz, J. V.; Cioslowski, J.; Fox, D. J. Gaussian, Inc., Wallingford CT, 2009. 


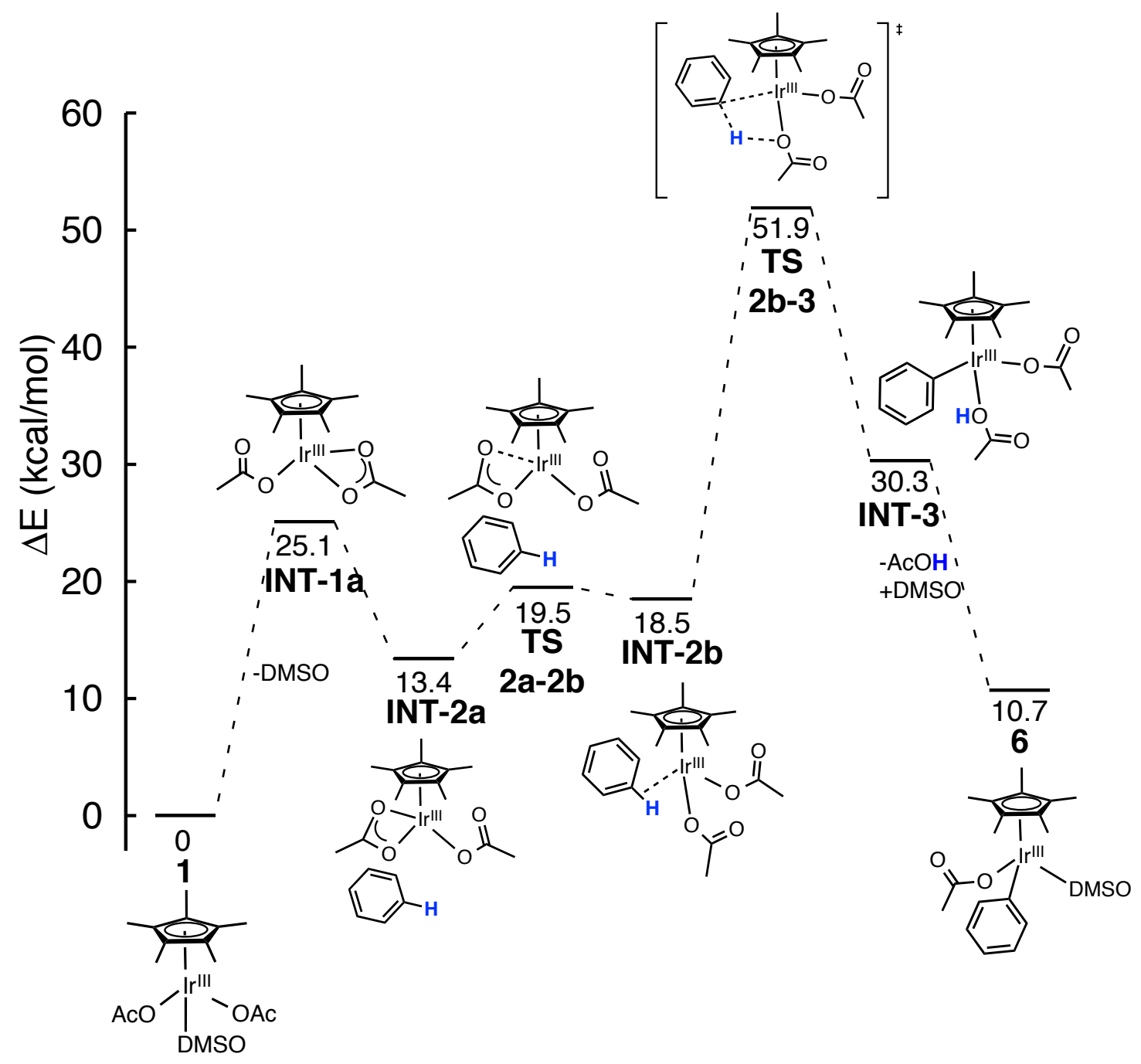

Figure S13. $\sigma$-bond metathesis pathway. Relative electronic energies of the intermediates and transition states along the $\mathrm{C}-\mathrm{H}$ sigma-bond metathesis pathway from $\mathbf{1}$ to $\mathbf{6}$ are shown. All optimizations were done using B3PW91-D3 functional with SDD $+\mathrm{f}$ for Ir and 6-31G* for $\mathrm{C}, \mathrm{H}, \mathrm{O}, \mathrm{S}$ in vacuum. 


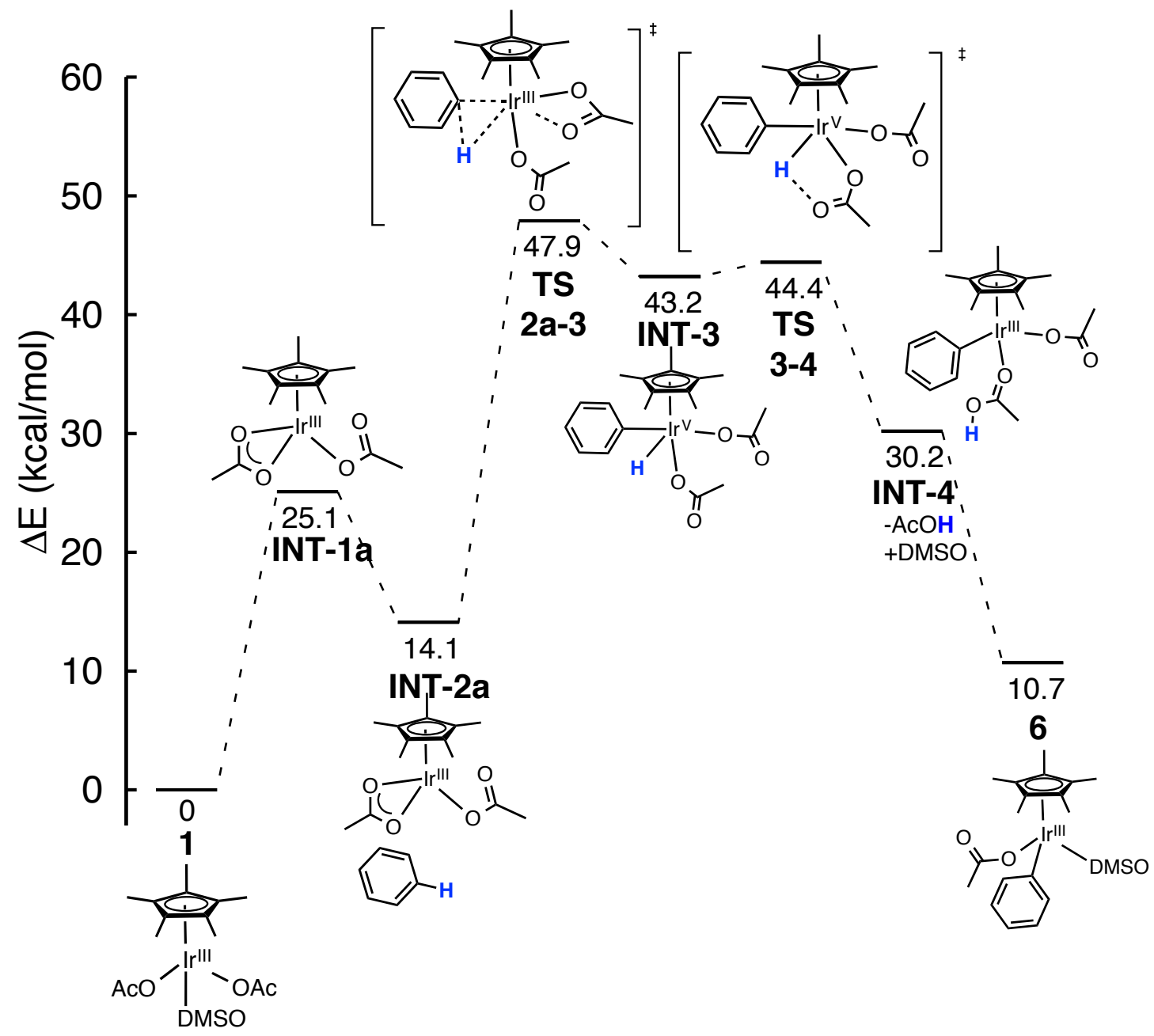

Figure S14. Oxidative addition pathway. Relative electronic energies of the intermediates and transition states along the oxidative addition pathway from $\mathbf{1}$ to $\mathbf{6}$ are shown. All optimizations were done using B3PW91-D3 functional with SDD $+\mathrm{f}$ for Ir and 6-31G* for $\mathrm{C}, \mathrm{H}, \mathrm{O}, \mathrm{S}$ in vacuum. 


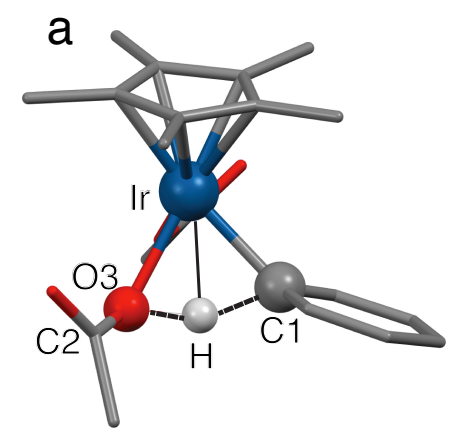

$\mathrm{H}-\mathrm{Ir}-\mathrm{O} 3-\mathrm{C} 2110^{\circ}$ $\mathrm{C} 1-\mathrm{Ir}-\mathrm{O} 3-\mathrm{H}-7.6^{\circ}$

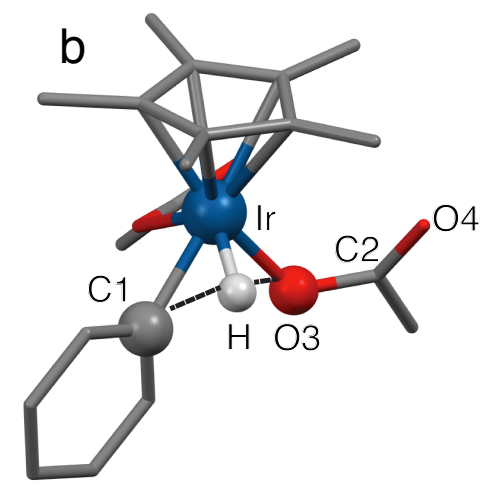

C1-Ir-O3-H $-47.28^{\circ}$

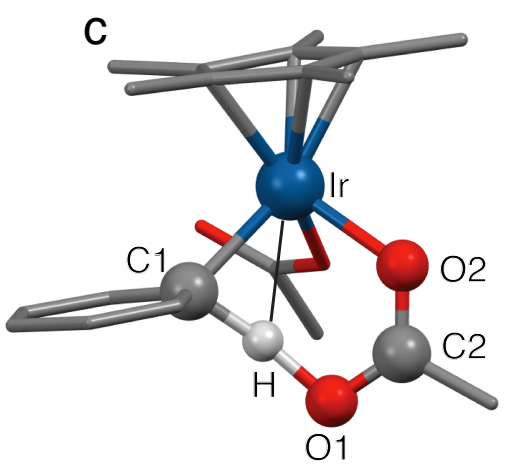

$\mathrm{H}-\mathrm{Ir}-\mathrm{O} 2-\mathrm{C} 216^{\circ}$

Figure S15. Transition state for the rate-determining step for the three mechanisms (a) $\sigma$ bond metathesis, (b) oxidative addition and (c) acetate-assisted.

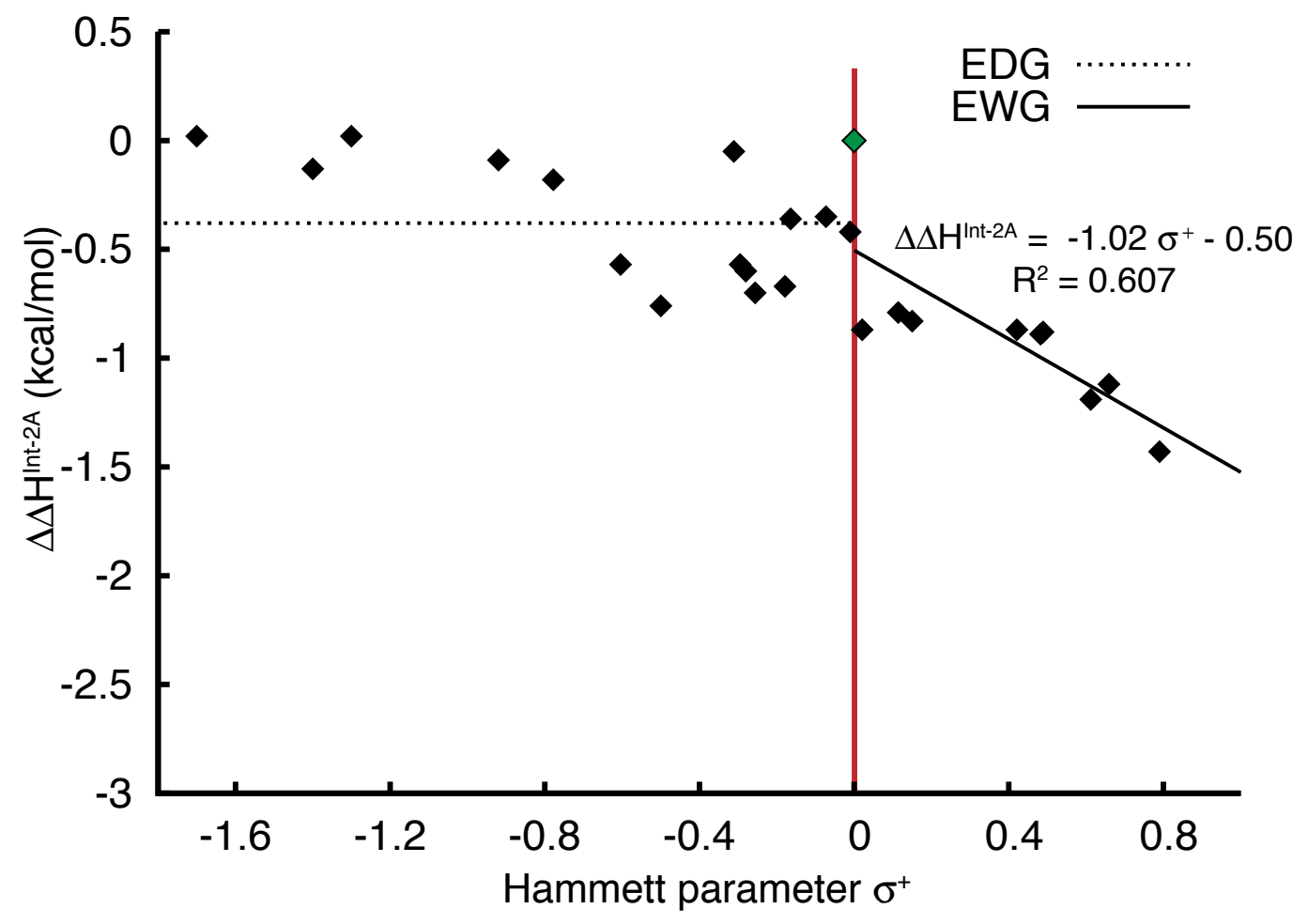

Figure S16. The change in enthalpy $(\Delta \Delta \mathrm{H})$ of Int-2a for the acetate-assisted pathway vs. the Hammett parameter of substituent $Y$ in para-functionalized benzene. Linear fits with the correlation coefficient, $\mathrm{R}^{2}$, are shown for EWGs. No correlation was observed for EDGs. The change in the enthalpy of activation $(\Delta \Delta \mathrm{H})$ is the enthalpy of functionalized benzene relative to the unsubstituted benzene. 


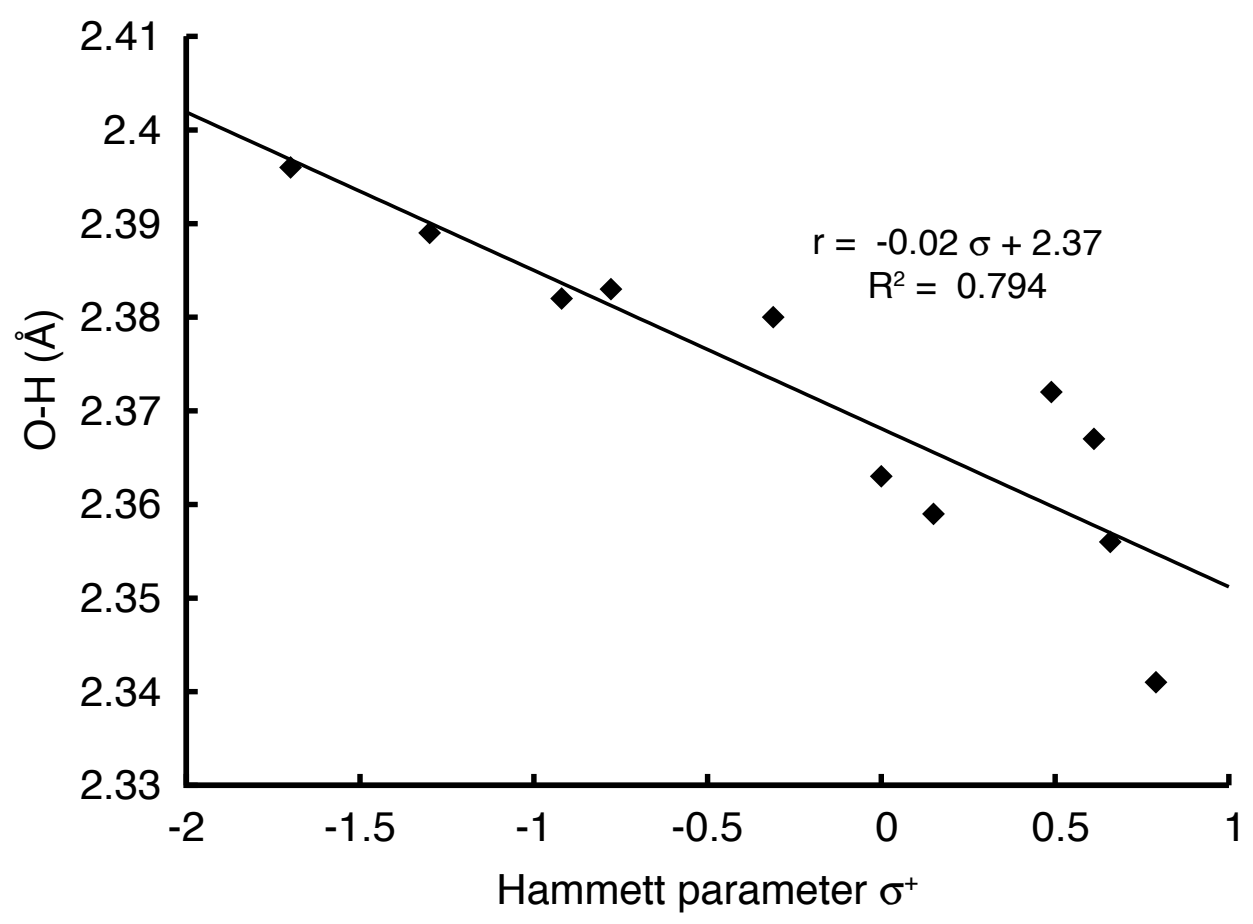

Figure S17. Plot of $\mathrm{O}$ (acetate) $-\mathrm{H}$ (arene) bond for Int-2a vs. $\sigma^{+}$.

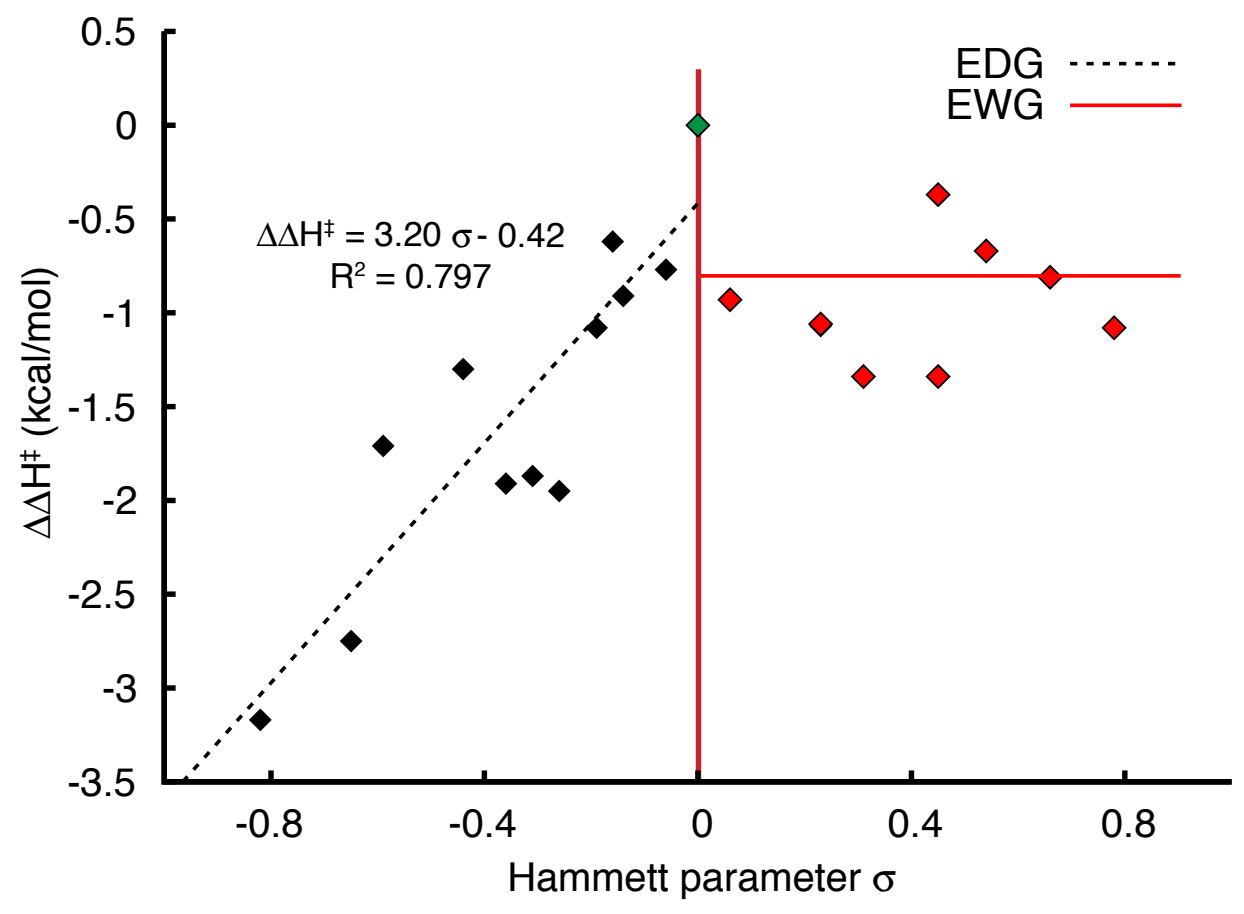

Figure S18. The change in enthalpy of activation $\left(\Delta \Delta H^{+}\right)$for the acetate-assisted pathway vs. the Hammett parameter of substituent $Y$ in para-functionalized benzene. Linear fits with the correlation coefficient, $\mathrm{R}^{2}$, are shown for EDGs. 


\section{X-RAY DATA}

\section{Crystal Structure Report for 8}

Crystal description. A pale yellow block-like specimen of $\mathrm{C}_{19} \mathrm{H}_{27} \mathrm{IrO}_{2} \mathrm{~S}$, approximate dimensions $0.098 \mathrm{~mm} \times 0.123 \mathrm{~mm} \times 0.245 \mathrm{~mm}$, was used for the X-ray crystallographic analysis. Instrument description The X-ray intensity data were measured.

Data collection The total exposure time was 12.09 hours. Integration The frames were integrated with the Bruker SAINT software package using a narrow-frame algorithm. The integration of the data using an orthorhombic unit cell yielded a total of 59442 reflections to a maximum $\theta$ angle of $36.38^{\circ}$ ( $0.60 \AA$ resolution), of which 8925 were independent (average redundancy 6.660 , completeness $=99.9 \%, \mathrm{R}_{\text {int }}=4.30 \%, \mathrm{R}_{\mathrm{sig}}=3.77 \%$ ) and 8360 (93.67\%) were greater than $2 \sigma\left(\mathrm{F}^{2}\right)$. Unit cell The final cell constants of $\underline{a}=9.1162(2) \AA$, $\underline{\mathrm{b}}=13.3585(3) \AA, \underline{\mathrm{c}}=15.0708(4) \AA$, volume $=1835.30(8) \AA^{3}$, are based upon the refinement of the XYZ-centroids of 275 reflections above $20 \sigma(\mathrm{I})$ with $5.229^{\circ}<2 \theta<$ $51.35^{\circ}$. Scaling Data were corrected for absorption effects using the numerical method (SADABS). The ratio of minimum to maximum apparent transmission was 0.498 . The calculated minimum and maximum transmission coefficients (based on crystal size) are 0.2640 and 0.5310 .

Structure solution Structure refinement The final anisotropic full-matrix least-squares refinement on $\mathrm{F}^{2}$ with 215 variables converged at $\mathrm{R} 1=2.38 \%$, for the observed data and $\mathrm{wR} 2=4.94 \%$ for all data. The goodness-of-fit was 1.032 . The largest peak in the final difference electron density synthesis was $3.434 \mathrm{e}^{-} / \AA^{3}$ and the largest hole was $-0.894 \mathrm{e}^{-}$ $/ \AA^{3}$ with an RMS deviation of $0.144 \mathrm{e}^{-} / \AA^{3}$. On the basis of the final model, the calculated density was $1.852 \mathrm{~g} / \mathrm{cm}^{3}$ and F(000), $1000 \mathrm{e}^{-}$. Structure Packing Sample and crystal data 
Table S1. Sample and crystal data for 8.

\begin{tabular}{|c|c|}
\hline Identification code & rds379 \\
\hline Chemical formula & $\mathrm{C}_{19} \mathrm{H}_{27} \mathrm{IrO}_{2} \mathrm{~S}$ \\
\hline Formula weight & $511.70 \mathrm{~g} / \mathrm{mol}$ \\
\hline Temperature & $100(2) \mathrm{K}$ \\
\hline Wavelength & $0.71073 \AA$ \\
\hline Crystal size & $0.098 \times 0.123 \times 0.245 \mathrm{~mm}$ \\
\hline Crystal habit & pale yellow block \\
\hline Crystal system & orthorhombic \\
\hline Space group & P 212121 \\
\hline \multirow[t]{3}{*}{ Unit cell dimensions } & $a=9.1162(2) \AA$ \\
\hline & $\mathrm{b}=13.3585(3) \AA \quad \beta=90^{\circ}$ \\
\hline & $\mathrm{c}=15.0708(4) \AA \quad \gamma=90^{\circ}$ \\
\hline Volume & $1835.30(8) \AA^{3}$ \\
\hline $\mathrm{Z}$ & 4 \\
\hline Density (calculated) & $1.852 \mathrm{~g} / \mathrm{cm}^{3}$ \\
\hline Absorption coefficient & $7.395 \mathrm{~mm}^{-1}$ \\
\hline $\mathrm{F}(000)$ & 1000 \\
\hline
\end{tabular}


Data collection and structure refinement

Table S2. Data collection and structure refinement for $\mathbf{8 .}$

Theta range for data collection

Index ranges

Reflections collected

Independent reflections

Coverage of independent reflections

Absorption correction

Max. and min. transmission

Refinement method

Refinement program

Function minimized

Data / restraints / parameters

Goodness-of-fit on $\mathrm{F}^{2}$

$\Delta / \sigma_{\max }$

Final R indices

Weighting scheme

Absolute structure parameter

Largest diff. peak and hole

R.M.S. deviation from mean
2.04 to $36.38^{\circ}$

$-13<=\mathrm{h}<=15,-22<=\mathrm{k}<=22,-25<=\mathrm{l}<=23$

59442

$8925[\mathrm{R}(\mathrm{int})=0.0430]$

$99.9 \%$

numerical

0.5310 and 0.2640

Full-matrix least-squares on $\mathrm{F}^{2}$

SHELXL-2013 (Sheldrick, 2013)

$\Sigma \mathrm{w}\left(\mathrm{F}_{\mathrm{o}}^{2}-\mathrm{F}_{\mathrm{c}}{ }^{2}\right)^{2}$

$8925 / 0 / 215$

1.032

0.003

8360 data; $\mathrm{I}>2 \sigma(\mathrm{I}) \quad \mathrm{R} 1=0.0238, \mathrm{wR} 2=0.0481$

all data $\quad \mathrm{R} 1=0.0278, \mathrm{wR} 2=0.0494$

$\mathrm{w}=1 /\left[\sigma^{2}\left(\mathrm{~F}_{\mathrm{o}}^{2}\right)+(0.0210 \mathrm{P})^{2}\right]$

where $\mathrm{P}=\left(\mathrm{F}_{\mathrm{o}}{ }^{2}+2 \mathrm{~F}_{\mathrm{c}}{ }^{2}\right) / 3$

$0.0(0)$

3.434 and $-0.894 \mathrm{e}^{-3}$

$0.144 \mathrm{e}^{-3}$ 
Atomic coordinates and equivalent isotropic displacement parameters

Table S3. Atomic coordinates and equivalent isotropic atomic displacement parameters $\left(\AA^{2}\right)$ for 8 . $\mathrm{U}(\mathrm{eq})$ is defined as one third of the trace of the orthogonalized $\mathrm{U}_{\mathrm{ij}}$ tensor.

\begin{tabular}{lllll}
\hline & $\mathbf{x} / \mathbf{a}$ & $\mathbf{y} / \mathbf{b}$ & $\mathbf{z} / \mathbf{c}$ & $\mathbf{U}(\mathbf{e q})$ \\
Ir1 & $0.65150(2)$ & $0.48252(2)$ & $0.89344(2)$ & $0.01185(3)$ \\
S1 & $0.56683(10)$ & $0.63285(6)$ & $0.93008(6)$ & $0.01513(15)$ \\
O1 & $0.3682(3)$ & $0.3915(2)$ & $0.9527(2)$ & $0.0255(6)$ \\
O2 & $0.6435(4)$ & $0.6866(2)$ & $0.00187(19)$ & $0.0297(6)$ \\
C1 & $0.8948(4)$ & $0.5036(3)$ & $0.9176(2)$ & $0.0171(7)$ \\
C2 & $0.8686(4)$ & $0.5145(3)$ & $0.8223(2)$ & $0.0184(6)$ \\
C3 & $0.8116(4)$ & $0.4225(3)$ & $0.7916(2)$ & $0.0164(6)$ \\
C4 & $0.7970(4)$ & $0.3553(3)$ & $0.8661(2)$ & $0.0171(6)$ \\
C5 & $0.8532(4)$ & $0.4056(3)$ & $0.9433(2)$ & $0.0173(6)$ \\
C6 & $0.9687(5)$ & $0.5788(3)$ & $0.9762(3)$ & $0.0256(8)$ \\
C7 & $0.9152(5)$ & $0.6025(3)$ & $0.7687(3)$ & $0.0287(9)$ \\
C8 & $0.7805(4)$ & $0.3949(3)$ & $0.6977(2)$ & $0.0243(8)$ \\
C9 & $0.7552(5)$ & $0.2464(3)$ & $0.8598(3)$ & $0.0240(8)$ \\
C10 & $0.8748(5)$ & $0.3607(3)$ & $0.0339(3)$ & $0.0261(8)$ \\
C11 & $0.4642(4)$ & $0.4600(3)$ & $0.8197(2)$ & $0.0153(6)$ \\
C12 & $0.3500(5)$ & $0.4124(3)$ & $0.8650(3)$ & $0.0201(6)$ \\
C13 & $0.2173(4)$ & $0.3892(3)$ & $0.8245(3)$ & $0.0269(8)$ \\
C14 & $0.1960(5)$ & $0.4175(4)$ & $0.7367(3)$ & $0.0305(10)$ \\
C15 & $0.3030(4)$ & $0.4685(3)$ & $0.6906(3)$ & $0.0277(9)$ \\
C16 & $0.4370(4)$ & $0.4884(3)$ & $0.7325(2)$ & $0.0200(6)$ \\
C17 & $0.5136(4)$ & $0.4157(3)$ & $0.9868(3)$ & $0.0232(8)$ \\
C18 & $0.3761(5)$ & $0.6375(3)$ & $0.9543(4)$ & $0.0320(10)$ \\
C19 & $0.5679(6)$ & $0.7153(3)$ & $0.8375(3)$ & $0.0321(10)$ \\
\hline
\end{tabular}


Bond lengths

Table S4. Bond lengths $(\AA)$ for 8 .

\begin{tabular}{llll} 
Ir1-C11 & $2.060(4)$ & Ir1-C17 & $2.087(4)$ \\
Ir1-C4 & $2.195(4)$ & Ir1-S1 & $2.2212(9)$ \\
Ir1-C5 & $2.236(4)$ & Ir1-C3 & $2.265(3)$ \\
Ir1-C1 & $2.266(3)$ & Ir1-C2 & $2.291(3)$ \\
S1-O2 & $1.475(3)$ & S1-C18 & $1.778(4)$ \\
S1-C19 & $1.778(4)$ & O1-C12 & $1.362(5)$ \\
O1-C17 & $1.458(5)$ & C1-C5 & $1.417(5)$ \\
C1-C2 & $1.463(5)$ & C1-C6 & $1.497(5)$ \\
C2-C3 & $1.412(5)$ & C2-C7 & $1.489(6)$ \\
C3-C4 & $1.443(5)$ & C3-C8 & $1.490(5)$ \\
C4-C5 & $1.439(5)$ & C4-C9 & $1.507(5)$ \\
C5-C10 & $1.504(5)$ & C6-H6A & 0.98 \\
C6-H6B & 0.98 & C6-H6C & 0.98 \\
C7-H7A & 0.98 & C7-H7B & 0.98 \\
C7-H7C & 0.98 & C8-H8A & 0.98 \\
C8-H8B & 0.98 & C8-H8C & 0.98 \\
C9-H9A & 0.98 & C9-H9B & 0.98 \\
C9-H9C & 0.98 & C10-H10A & 0.98 \\
C10-H10B & 0.98 & C10-H10C & 0.98 \\
C11-C16 & $1.389(5)$ & C11-C12 & $1.398(5)$ \\
C12-C13 & $1.390(6)$ & C13-C14 & $1.390(7)$ \\
C13-H13 & 0.95 & C14-C15 & $1.378(7)$ \\
C14-H14 & 0.95 & C15-C16 & $1.401(5)$ \\
C15-H15 & 0.95 & C16-H16 & 0.95 \\
C17-H17A & 0.99 & C17-H17B & 0.99 \\
C18-H18A & 0.98 & C18-H18B & 0.98 \\
C18-H18C & 0.98 & C19-H19A & 0.98 \\
C19-H19B & 0.98 & C19-H19C & 0.98 \\
\hline
\end{tabular}


Bond angles

Table S5. Bond angles $\left({ }^{\circ}\right)$ for 8.

$\begin{array}{llll}\text { C11-Ir1-C17 } & 78.59(15) & \text { C11-Ir1-C4 } & 106.65(13) \\ \text { C17-Ir1-C4 } & 99.19(15) & \text { C11-Ir1-S1 } & 88.75(10) \\ \text { C17-Ir1-S1 } & 90.55(12) & \text { C4-Ir1-S1 } & 163.08(10) \\ \text { C11-Ir1-C5 } & 142.74(13) & \text { C17-Ir1-C5 } & 94.13(15) \\ \text { C4-Ir1-C5 } & 37.88(13) & \text { S1-Ir1-C5 } & 128.13(9) \\ \text { C11-Ir1-C3 } & 96.71(14) & \text { C17-Ir1-C3 } & 133.92(16) \\ \text { C4-Ir1-C3 } & 37.71(13) & \text { S1-Ir1-C3 } & 135.44(10) \\ \text { C5-Ir1-C3 } & 62.31(13) & \text { C11-Ir1-C1 } & 156.45(14) \\ \text { C17-Ir1-C1 } & 122.30(15) & \text { C4-Ir1-C1 } & 62.26(13) \\ \text { S1-Ir1-C1 } & 100.84(9) & \text { C5-Ir1-C1 } & 36.68(13) \\ \text { C3-Ir1-C1 } & 61.43(13) & \text { C11-Ir1-C2 } & 119.40(14) \\ \text { C17-Ir1-C2 } & 156.26(15) & \text { C4-Ir1-C2 } & 62.23(14) \\ \text { S1-Ir1-C2 } & 104.37(11) & \text { C5-Ir1-C2 } & 62.14(14) \\ \text { C3-Ir1-C2 } & 36.10(14) & \text { C1-Ir1-C2 } & 37.46(13) \\ \text { O2-S1-C18 } & 107.2(2) & \text { O2-S1-C19 } & 105.8(2) \\ \text { C18-S1-C19 } & 98.3(2) & \text { O2-S1-Ir1 } & 117.25(13) \\ \text { C18-S1-Ir1 } & 114.99(15) & \text { C19-S1-Ir1 } & 111.29(16) \\ \text { C12-O1-C17 } & 114.0(3) & \text { C5-C1-C2 } & 108.5(3) \\ \text { C5-C1-C6 } & 125.4(3) & \text { C2-C1-C6 } & 125.8(4) \\ \text { C5-C1-Ir1 } & 70.5(2) & \text { C2-C1-Ir1 } & 72.21(18) \\ \text { C6-C1-Ir1 } & 128.2(3) & \text { C3-C2-C1 } & 107.2(3) \\ \text { C3-C2-C7 } & 127.9(4) & \text { C1-C2-C7 } & 124.4(4) \\ \text { C3-C2-Ir1 } & 70.9(2) & \text { C1-C2-Ir1 } & 70.33(18) \\ \text { C7-C2-Ir1 } & 130.4(3) & \text { C2-C3-C4 } & 108.7(3) \\ \text { C2-C3-C8 } & 126.7(4) & \text { C4-C3-C8 } & 124.5(3) \\ \text { C2-C3-Ir1 } & 73.0(2) & \text { C4-C3-Ir1 } & 68.50(19) \\ \text { C8-C3-Ir1 } & 127.5(3) & \text { C5-C4-C3 } & 107.8(3) \\ \text { C5-C4-C9 } & 126.3(3) & \text { C3-C4-C9 } & 125.1(3) \\ \text { C5-C4-Ir1 } & 72.6(2) & \text { C3-C4-Ir1 } & 73.8(2) \\ \text { C9-C4-Ir1 } & 127.3(3) & \text { C1-C5-C4 } & 107.8(3) \\ \text { C1-C5-C10 } & 125.6(3) & \text { C4-C5-C10 } & 126.5(3) \\ \text { C1-C5-Ir1 } & 72.8(2) & \text { C4-C5-Ir1 } & 69.5(2) \\ \text { C10-C5-Ir1 } & 126.6(3) & \text { C1-C6-H6A } & 109.5 \\ \text { C1-C6-H6B } & 109.5 & \text { H6A-C6-H6B } & 109.5 \\ \text { C1-C6-H6C } & 109.5 & \text { H6A-C6-H6C } & 109.5 \\ \text { H6B-C6-H6C } & 109.5 & \text { C2-C7-H7A } & 109.5 \\ \text { C2-C7-H7B } & 109.5 & \text { H7A-C7-H7B } & 109.5 \\ \text { C2-C7-H7C } & 109.5 & \text { H7A-C7-H7C } & 109.5 \\ \text { H7B-C7-H7C } & 109.5 & \text { C3-C8-H8A } & 109.5 \\ \text { C3-C8-H8B } & 109.5 & \text { H8A-C8-H8B } & 109.5 \\ \text { C3-C8-H8C } & 109.5 & \text { H8A-C8-H8C } & 109.5 \\ \text { C1 } 13 & \end{array}$




\begin{tabular}{llll} 
H8B-C8-H8C & 109.5 & C4-C9-H9A & 109.5 \\
C4-C9-H9B & 109.5 & H9A-C9-H9B & 109.5 \\
C4-C9-H9C & 109.5 & H9A-C9-H9C & 109.5 \\
H9B-C9-H9C & 109.5 & C5-C10-H10A & 109.5 \\
C5-C10-H10B & 109.5 & H10A-C10-H10B & 109.5 \\
C5-C10-H10C & 109.5 & H10A-C10-H10C & 109.5 \\
H10B-C10-H10C & 109.5 & C16-C11-C12 & $117.0(3)$ \\
C16-C11-Ir1 & $128.2(3)$ & C12-C11-Ir1 & $114.8(3)$ \\
O1-C12-C13 & $119.1(4)$ & O1-C12-C11 & $118.5(4)$ \\
C13-C12-C11 & $122.3(4)$ & C14-C13-C12 & $118.6(4)$ \\
C14-C13-H13 & 120.7 & C12-C13-H13 & 120.7 \\
C15-C14-C13 & $121.0(4)$ & C15-C14-H14 & 119.5 \\
C13-C14-H14 & 119.5 & C14-C15-C16 & $118.9(4)$ \\
C14-C15-H15 & 120.5 & C16-C15-H15 & 120.5 \\
C11-C16-C15 & $122.0(4)$ & C11-C16-H16 & 119.0 \\
C15-C16-H16 & 119.0 & O1-C17-Ir1 & $113.9(3)$ \\
O1-C17-H17A & 108.8 & Ir1-C17-H17A & 108.8 \\
O1-C17-H17B & 108.8 & Ir1-C17-H17B & 108.8 \\
H17A-C17-H17B & 107.7 & S1-C18-H18A & 109.5 \\
S1-C18-H18B & 109.5 & H18A-C18-H18B & 109.5 \\
S1-C18-H18C & 109.5 & H18A-C18-H18C & 109.5 \\
H18B-C18-H18C & 109.5 & S1-C19-H19A & 109.5 \\
S1-C19-H19B & 109.5 & H19A-C19-H19B & 109.5 \\
S1-C19-H19C & 109.5 & H19A-C19-H19C & 109.5 \\
H19B-C19-H19C & 109.5 & & \\
\hline
\end{tabular}


Torsion angles

Table S6. Torsion angles $\left({ }^{\circ}\right)$ for 8.

\begin{tabular}{llll} 
C5-C1-C2-C3 & $-0.2(4)$ & C6-C1-C2-C3 & $173.5(3)$ \\
Ir1-C1-C2-C3 & $-61.7(2)$ & C5-C1-C2-C7 & $-172.4(4)$ \\
C6-C1-C2-C7 & $1.3(6)$ & Ir1-C1-C2-C7 & $126.1(4)$ \\
C5-C1-C2-Ir1 & $61.6(3)$ & C6-C1-C2-Ir1 & $-124.7(4)$ \\
C1-C2-C3-C4 & $1.9(4)$ & C7-C2-C3-C4 & $173.7(4)$ \\
Ir1-C2-C3-C4 & $-59.5(2)$ & C1-C2-C3-C8 & $-174.2(3)$ \\
C7-C2-C3-C8 & $-2.4(6)$ & Ir1-C2-C3-C8 & $124.4(4)$ \\
C1-C2-C3-Ir1 & $61.3(2)$ & C7-C2-C3-Ir1 & $-126.8(4)$ \\
C2-C3-C4-C5 & $-2.9(4)$ & C8-C3-C4-C5 & $173.3(3)$ \\
Ir1-C3-C4-C5 & $-65.2(3)$ & C2-C3-C4-C9 & $-173.2(4)$ \\
C8-C3-C4-C9 & $3.1(6)$ & Ir1-C3-C4-C9 & $124.6(4)$ \\
C2-C3-C4-Ir1 & $62.2(2)$ & C8-C3-C4-Ir1 & $-121.5(3)$ \\
C2-C1-C5-C4 & $-1.6(4)$ & C6-C1-C5-C4 & $-175.4(3)$ \\
Ir1-C1-C5-C4 & $61.0(3)$ & C2-C1-C5-C10 & $174.3(3)$ \\
C6-C1-C5-C10 & $0.5(6)$ & Ir1-C1-C5-C10 & $-123.1(4)$ \\
C2-C1-C5-Ir1 & $-62.6(2)$ & C6-C1-C5-Ir1 & $123.6(4)$ \\
C3-C4-C5-C1 & $2.8(4)$ & C9-C4-C5-C1 & $172.9(4)$ \\
Ir1-C4-C5-C1 & $-63.1(3)$ & C3-C4-C5-C10 & $-173.1(4)$ \\
C9-C4-C5-C10 & $-2.9(6)$ & Ir1-C4-C5-C10 & $121.0(4)$ \\
C3-C4-C5-Ir1 & $65.9(2)$ & C9-C4-C5-Ir1 & $-124.0(4)$ \\
C17-O1-C12-C13 & $177.8(3)$ & C17-O1-C12-C11 & $-4.6(5)$ \\
C16-C11-C12-O1 & $-174.2(3)$ & Ir1-C11-C12-O1 & $4.1(4)$ \\
C16-C11-C12-C13 & $3.3(5)$ & Ir1-C11-C12-C13 & $-178.4(3)$ \\
O1-C12-C13-C14 & $175.3(4)$ & C11-C12-C13-C14 & $-2.2(6)$ \\
C12-C13-C14-C15 & $-0.7(6)$ & C13-C14-C15-C16 & $2.4(6)$ \\
C12-C11-C16-C15 & $-1.5(6)$ & Ir1-C11-C16-C15 & $-179.6(3)$ \\
C14-C15-C16-C11 & $-1.2(6)$ & C12-O1-C17-Ir1 & $2.9(4)$ \\
\hline
\end{tabular}


Anisotropic displacement parameters

Table S7. Anisotropic atomic displacement parameters $\left(\AA^{2}\right)$ for $\mathbf{8}$.

The anisotropic atomic displacement factor exponent takes the form: $-2 \pi^{2}\left[\mathrm{~h}^{2} \mathrm{a}^{* 2} \mathrm{U}_{11}+\ldots\right.$ $+2 \mathrm{~h} \mathrm{k} \mathrm{a}^{*} \mathrm{~b}^{*} \mathrm{U}_{12}$ ]

\begin{tabular}{lllllll} 
& $\mathbf{U}_{11}$ & $\mathbf{U}_{\mathbf{2 2}}$ & $\mathbf{U}_{\mathbf{3 3}}$ & $\mathbf{U}_{\mathbf{2 3}}$ & $\mathbf{U}_{\mathbf{1 3}}$ & $\mathbf{U}_{\mathbf{1 2}}$ \\
$\mathrm{Ir} 1$ & $0.00919(5)$ & $0.00909(4)$ & $0.01728(5)$ & $0.00035(5)$ & $0.00067(5)$ & $0.00012(5)$ \\
$\mathrm{S} 1$ & $0.0121(4)$ & $0.0114(3)$ & $0.0219(4)$ & $-0.0024(3)$ & $-0.0004(3)$ & $0.0013(3)$ \\
$\mathrm{O} 1$ & $0.0158(15)$ & $0.0264(15)$ & $0.0342(15)$ & $0.0089(12)$ & $0.0046(12)$ & $-0.0046(12)$ \\
$\mathrm{O} 2$ & $0.0314(16)$ & $0.0237(13)$ & $0.0341(14)$ & $-0.0130(11)$ & $-0.0114(13)$ & $0.0047(14)$ \\
$\mathrm{C} 1$ & $0.0091(13)$ & $0.0161(17)$ & $0.0261(15)$ & $-0.0035(11)$ & $-0.0006(11)$ & $0.0017(11)$ \\
$\mathrm{C} 2$ & $0.0123(15)$ & $0.0197(14)$ & $0.0233(14)$ & $-0.0001(16)$ & $0.0051(12)$ & $0.0024(14)$ \\
$\mathrm{C} 3$ & $0.0094(14)$ & $0.0176(15)$ & $0.0222(15)$ & $-0.0028(12)$ & $0.0024(11)$ & $0.0011(12)$ \\
$\mathrm{C} 4$ & $0.0134(15)$ & $0.0150(15)$ & $0.0229(15)$ & $-0.0042(12)$ & $0.0001(12)$ & $0.0024(12)$ \\
C5 & $0.0141(15)$ & $0.0150(13)$ & $0.0228(14)$ & $-0.0020(11)$ & $-0.0026(14)$ & $0.0050(15)$ \\
C6 & $0.0168(18)$ & $0.0227(18)$ & $0.037(2)$ & $-0.0114(16)$ & $-0.0018(16)$ & $-0.0031(15)$ \\
C7 & $0.022(2)$ & $0.025(2)$ & $0.039(2)$ & $0.0061(17)$ & $0.0093(17)$ & $-0.0042(16)$ \\
C8 & $0.0178(18)$ & $0.034(2)$ & $0.0213(17)$ & $-0.0046(15)$ & $0.0026(13)$ & $0.0017(16)$ \\
C9 & $0.026(2)$ & $0.0134(15)$ & $0.0331(19)$ & $-0.0045(14)$ & $-0.0016(16)$ & $0.0000(15)$ \\
C10 & $0.030(2)$ & $0.0246(18)$ & $0.0240(17)$ & $0.0018(14)$ & $-0.0065(15)$ & $0.0066(16)$ \\
C11 & $0.0087(13)$ & $0.0120(13)$ & $0.0251(14)$ & $-0.0054(12)$ & $0.0008(12)$ & $-0.0007(11)$ \\
C12 & $0.0151(15)$ & $0.0121(13)$ & $0.0331(16)$ & $-0.0022(12)$ & $0.0031(16)$ & $-0.0026(15)$ \\
C13 & $0.0108(17)$ & $0.0216(18)$ & $0.048(2)$ & $-0.0095(18)$ & $0.0051(16)$ & $-0.0040(14)$ \\
C14 & $0.0132(17)$ & $0.032(2)$ & $0.046(2)$ & $-0.0200(19)$ & $-0.0056(16)$ & $0.0025(16)$ \\
C15 & $0.0204(17)$ & $0.033(2)$ & $0.0298(18)$ & $-0.0120(17)$ & $-0.0076(14)$ & $0.0056(16)$ \\
C16 & $0.0134(15)$ & $0.0224(16)$ & $0.0242(15)$ & $-0.0065(14)$ & $-0.0017(11)$ & $0.0008(15)$ \\
C17 & $0.0188(18)$ & $0.0203(17)$ & $0.0303(19)$ & $0.0066(14)$ & $0.0044(14)$ & $-0.0004(15)$ \\
C18 & $0.018(2)$ & $0.0208(19)$ & $0.057(3)$ & $-0.0101(19)$ & $0.0109(18)$ & $0.0027(15)$ \\
C19 & $0.040(3)$ & $0.0190(18)$ & $0.037(2)$ & $0.0077(16)$ & $0.0017(19)$ & $0.0072(19)$ \\
\hline
\end{tabular}


Hydrogen atom coordinates and isotropic atomic displacement parameters

Table S8. Hydrogen atomic coordinates and isotropic atomic displacement parameters $\left(\AA^{2}\right)$ for 8.

\begin{tabular}{lllll} 
& $\mathbf{x} / \mathbf{a}$ & $\mathbf{y} / \mathbf{b}$ & $\mathbf{z} / \mathbf{c}$ & $\mathbf{U}(\mathbf{e q})$ \\
H6A & 0.9188 & 0.5811 & 1.0337 & 0.038 \\
H6B & 0.9643 & 0.6450 & 0.9482 & 0.038 \\
H6C & 1.0714 & 0.5596 & 0.9849 & 0.038 \\
H7A & 1.0157 & 0.5922 & 0.7474 & 0.043 \\
H7B & 0.9114 & 0.6630 & 0.8055 & 0.043 \\
H7C & 0.8492 & 0.6103 & 0.7178 & 0.043 \\
H8A & 0.7860 & 0.4548 & 0.6603 & 0.037 \\
H8B & 0.6820 & 0.3658 & 0.6935 & 0.037 \\
H8C & 0.8530 & 0.3458 & 0.6774 & 0.037 \\
H9A & 0.8427 & 0.2064 & 0.8470 & 0.036 \\
H9B & 0.6833 & 0.2375 & 0.8121 & 0.036 \\
H9C & 0.7123 & 0.2246 & 0.9162 & 0.036 \\
H10A & 0.9787 & 0.3442 & 1.0423 & 0.039 \\
H10B & 0.8158 & 0.2996 & 1.0392 & 0.039 \\
H10C & 0.8441 & 0.4089 & 1.0793 & 0.039 \\
H13 & 0.1427 & 0.3547 & 0.8561 & 0.032 \\
H14 & 0.1062 & 0.4014 & 0.7080 & 0.037 \\
H15 & 0.2862 & 0.4898 & 0.6313 & 0.033 \\
H16 & 0.5116 & 0.5224 & 0.7004 & 0.024 \\
H17A & 0.5604 & 0.3535 & 1.0085 & 0.028 \\
H17B & 0.5029 & 0.4615 & 1.0381 & 0.028 \\
H18A & 0.3569 & 0.6017 & 1.0098 & 0.048 \\
H18B & 0.3211 & 0.6060 & 0.9059 & 0.048 \\
H18C & 0.3453 & 0.7074 & 0.9604 & 0.048 \\
H19A & 0.5311 & 0.7811 & 0.8557 & 0.048 \\
H19B & 0.5049 & 0.6882 & 0.7906 & 0.048 \\
H19C & 0.6684 & 0.7221 & 0.8151 & 0.048 \\
\hline
\end{tabular}




\section{Crystal Structure Report for 9}

Crystal description

A pale yellow block-like specimen of $\mathrm{C}_{19} \mathrm{H}_{27} \mathrm{IrO}_{2} \mathrm{~S}$, approximate dimensions $0.098 \mathrm{~mm} \mathrm{x}$ $0.123 \mathrm{~mm} \times 0.245 \mathrm{~mm}$, was used for the X-ray crystallographic analysis. Instrument description The X-ray intensity data were measured.

Data collection The total exposure time was 12.09 hours. Integration The frames were integrated with the Bruker SAINT software package using a narrow-frame algorithm. The integration of the data using an orthorhombic unit cell yielded a total of 59442 reflections to a maximum $\theta$ angle of $36.38^{\circ}(0.60 \AA$ resolution $)$, of which 8925 were independent (average redundancy 6.660 , completeness $=99.9 \%, \mathrm{R}_{\text {int }}=4.30 \%, \mathrm{R}_{\text {sig }}=3.77 \%$ ) and 8360 $(93.67 \%)$ were greater than $2 \sigma\left(\mathrm{F}^{2}\right)$. Unit cell The final cell constants of $\underline{a}=9.1162(2) \AA$, $\underline{\mathrm{b}}=13.3585(3) \AA, \underline{\mathrm{c}}=15.0708(4) \AA$, volume $=1835.30(8) \AA^{3}$, are based upon the refinement of the XYZ-centroids of 275 reflections above $20 \sigma(\mathrm{I})$ with $5.229^{\circ}<2 \theta<$ $51.35^{\circ}$. Scaling Data were corrected for absorption effects using the numerical method (SADABS). The ratio of minimum to maximum apparent transmission was 0.498 . The calculated minimum and maximum transmission coefficients (based on crystal size) are 0.2640 and 0.5310 .

Structure solution Structure refinement The final anisotropic full-matrix least-squares refinement on $\mathrm{F}^{2}$ with 215 variables converged at $\mathrm{R} 1=2.38 \%$, for the observed data and $\mathrm{wR} 2=4.94 \%$ for all data. The goodness-of-fit was 1.032 . The largest peak in the final difference electron density synthesis was $3.434 \mathrm{e}^{-} / \AA^{3}$ and the largest hole was $-0.894 \mathrm{e}^{-}$ $/ \AA^{3}$ with an RMS deviation of $0.144 \mathrm{e}^{-} / \AA^{3}$. On the basis of the final model, the calculated density was $1.852 \mathrm{~g} / \mathrm{cm}^{3}$ and F(000), $1000 \mathrm{e}^{-}$. Structure Packing Sample and crystal data 
Table S9. Sample and crystal data for 9.

\begin{tabular}{|c|c|}
\hline Identification code & rds379 \\
\hline Chemical formula & $\mathrm{C}_{19} \mathrm{H}_{27} \mathrm{IrO}_{2} \mathrm{~S}$ \\
\hline Formula weight & $511.70 \mathrm{~g} / \mathrm{mol}$ \\
\hline Temperature & $100(2) \mathrm{K}$ \\
\hline Wavelength & $0.71073 \AA$ \\
\hline Crystal size & $0.098 \times 0.123 \times 0.245 \mathrm{~mm}$ \\
\hline Crystal habit & pale yellow block \\
\hline Crystal system & orthorhombic \\
\hline Space group & P 212121 \\
\hline \multirow[t]{3}{*}{ Unit cell dimensions } & $a=9.1162(2) \AA$ \\
\hline & $\mathrm{b}=13.3585(3) \AA \quad \beta=90^{\circ}$ \\
\hline & $\mathrm{c}=15.0708(4) \AA \quad \gamma=90^{\circ}$ \\
\hline Volume & $1835.30(8) \AA^{3}$ \\
\hline $\mathrm{Z}$ & 4 \\
\hline Density (calculated) & $1.852 \mathrm{~g} / \mathrm{cm}^{3}$ \\
\hline Absorption coefficient & $7.395 \mathrm{~mm}^{-1}$ \\
\hline $\mathrm{F}(000)$ & 1000 \\
\hline
\end{tabular}


Data collection and structure refinement

Table S10. Data collection and structure refinement for 9.

Theta range for data collection

Index ranges

Reflections collected

Independent reflections

Coverage of independent reflections

Absorption correction

Max. and min. transmission

Refinement method

Refinement program

Function minimized

Data / restraints / parameters

Goodness-of-fit on $\mathrm{F}^{2}$

$\Delta / \sigma_{\max }$

Final $\mathrm{R}$ indices

Weighting scheme

Absolute structure parameter

Largest diff. peak and hole

R.M.S. deviation from mean
2.04 to $36.38^{\circ}$

$-13<=\mathrm{h}<=15,-22<=\mathrm{k}<=22,-25<=\mathrm{l}<=23$

59442

$8925[\mathrm{R}(\mathrm{int})=0.0430]$

$99.9 \%$

numerical

0.5310 and 0.2640

Full-matrix least-squares on $\mathrm{F}^{2}$

SHELXL-2013 (Sheldrick, 2013)

$\Sigma \mathrm{w}\left(\mathrm{F}_{\mathrm{o}}^{2}-\mathrm{F}_{\mathrm{c}}{ }^{2}\right)^{2}$

$8925 / 0 / 215$

1.032

0.003

8360 data; $\mathrm{I}>2 \sigma(\mathrm{I}) \quad \mathrm{R} 1=0.0238, \mathrm{wR} 2=0.0481$

all data $\quad \mathrm{R} 1=0.0278, \mathrm{wR} 2=0.0494$

$\mathrm{w}=1 /\left[\sigma^{2}\left(\mathrm{~F}_{\mathrm{o}}{ }^{2}\right)+(0.0210 \mathrm{P})^{2}\right]$

where $\mathrm{P}=\left(\mathrm{F}_{\mathrm{o}}{ }^{2}+2 \mathrm{~F}_{\mathrm{c}}{ }^{2}\right) / 3$

$0.0(0)$

3.434 and $-0.894 \mathrm{e}^{-3}$

$0.144 \mathrm{e}^{-3}$ 
Atomic coordinates and equivalent isotropic displacement parameters

Table S11. Atomic coordinates and equivalent isotropic atomic displacement parameters $\left(\AA^{2}\right)$ for 9.

$\mathrm{U}(\mathrm{eq})$ is defined as one third of the trace of the orthogonalized $\mathrm{U}_{\mathrm{ij}}$ tensor.

\begin{tabular}{lllll}
\hline & $\mathbf{x} / \mathbf{a}$ & $\mathbf{y} / \mathbf{b}$ & $\mathbf{z} / \mathbf{c}$ & $\mathbf{U}(\mathbf{e q})$ \\
Ir1 & $0.65150(2)$ & $0.48252(2)$ & $0.89344(2)$ & $0.01185(3)$ \\
S1 & $0.56683(10)$ & $0.63285(6)$ & $0.93008(6)$ & $0.01513(15)$ \\
O1 & $0.3682(3)$ & $0.3915(2)$ & $0.9527(2)$ & $0.0255(6)$ \\
O2 & $0.6435(4)$ & $0.6866(2)$ & $0.00187(19)$ & $0.0297(6)$ \\
C1 & $0.8948(4)$ & $0.5036(3)$ & $0.9176(2)$ & $0.0171(7)$ \\
C2 & $0.8686(4)$ & $0.5145(3)$ & $0.8223(2)$ & $0.0184(6)$ \\
C3 & $0.8116(4)$ & $0.4225(3)$ & $0.7916(2)$ & $0.0164(6)$ \\
C4 & $0.7970(4)$ & $0.3553(3)$ & $0.8661(2)$ & $0.0171(6)$ \\
C5 & $0.8532(4)$ & $0.4056(3)$ & $0.9433(2)$ & $0.0173(6)$ \\
C6 & $0.9687(5)$ & $0.5788(3)$ & $0.9762(3)$ & $0.0256(8)$ \\
C7 & $0.9152(5)$ & $0.6025(3)$ & $0.7687(3)$ & $0.0287(9)$ \\
C8 & $0.7805(4)$ & $0.3949(3)$ & $0.6977(2)$ & $0.0243(8)$ \\
C9 & $0.7552(5)$ & $0.2464(3)$ & $0.8598(3)$ & $0.0240(8)$ \\
C10 & $0.8748(5)$ & $0.3607(3)$ & $0.0339(3)$ & $0.0261(8)$ \\
C11 & $0.4642(4)$ & $0.4600(3)$ & $0.8197(2)$ & $0.0153(6)$ \\
C12 & $0.3500(5)$ & $0.4124(3)$ & $0.8650(3)$ & $0.0201(6)$ \\
C13 & $0.2173(4)$ & $0.3892(3)$ & $0.8245(3)$ & $0.0269(8)$ \\
C14 & $0.1960(5)$ & $0.4175(4)$ & $0.7367(3)$ & $0.0305(10)$ \\
C15 & $0.3030(4)$ & $0.4685(3)$ & $0.6906(3)$ & $0.0277(9)$ \\
C16 & $0.4370(4)$ & $0.4884(3)$ & $0.7325(2)$ & $0.0200(6)$ \\
C17 & $0.5136(4)$ & $0.4157(3)$ & $0.9868(3)$ & $0.0232(8)$ \\
C18 & $0.3761(5)$ & $0.6375(3)$ & $0.9543(4)$ & $0.0320(10)$ \\
C19 & $0.5679(6)$ & $0.7153(3)$ & $0.8375(3)$ & $0.0321(10)$ \\
\hline
\end{tabular}


Bond lengths

Table S12. Bond lengths ( $\AA$ ) for 9.

\begin{tabular}{llll} 
Ir1-C11 & $2.060(4)$ & Ir1-C17 & $2.087(4)$ \\
Ir1-C4 & $2.195(4)$ & Ir1-S1 & $2.2212(9)$ \\
Ir1-C5 & $2.236(4)$ & Ir1-C3 & $2.265(3)$ \\
Ir1-C1 & $2.266(3)$ & Ir1-C2 & $2.291(3)$ \\
S1-O2 & $1.475(3)$ & S1-C18 & $1.778(4)$ \\
S1-C19 & $1.778(4)$ & O1-C12 & $1.362(5)$ \\
O1-C17 & $1.458(5)$ & C1-C5 & $1.417(5)$ \\
C1-C2 & $1.463(5)$ & C1-C6 & $1.497(5)$ \\
C2-C3 & $1.412(5)$ & C2-C7 & $1.489(6)$ \\
C3-C4 & $1.443(5)$ & C3-C8 & $1.490(5)$ \\
C4-C5 & $1.439(5)$ & C4-C9 & $1.507(5)$ \\
C5-C10 & $1.504(5)$ & C6-H6A & 0.98 \\
C6-H6B & 0.98 & C6-H6C & 0.98 \\
C7-H7A & 0.98 & C7-H7B & 0.98 \\
C7-H7C & 0.98 & C8-H8A & 0.98 \\
C8-H8B & 0.98 & C8-H8C & 0.98 \\
C9-H9A & 0.98 & C9-H9B & 0.98 \\
C9-H9C & 0.98 & C10-H10A & 0.98 \\
C10-H10B & 0.98 & C10-H10C & 0.98 \\
C11-C16 & $1.389(5)$ & C11-C12 & $1.398(5)$ \\
C12-C13 & $1.390(6)$ & C13-C14 & $1.390(7)$ \\
C13-H13 & 0.95 & C14-C15 & $1.378(7)$ \\
C14-H14 & 0.95 & C15-C16 & $1.401(5)$ \\
C15-H15 & 0.95 & C16-H16 & 0.95 \\
C17-H17A & 0.99 & C17-H17B & 0.99 \\
C18-H18A & 0.98 & C18-H18B & 0.98 \\
C18-H18C & 0.98 & C19-H19A & 0.98 \\
C19-H19B & 0.98 & C19-H19C & 0.98 \\
\hline
\end{tabular}


Bond angles

Table S13. Bond angles $\left({ }^{\circ}\right)$ for 9.

$\begin{array}{llll}\text { C11-Ir1-C17 } & 78.59(15) & \text { C11-Ir1-C4 } & 106.65(13) \\ \text { C17-Ir1-C4 } & 99.19(15) & \text { C11-Ir1-S1 } & 88.75(10) \\ \text { C17-Ir1-S1 } & 90.55(12) & \text { C4-Ir1-S1 } & 163.08(10) \\ \text { C11-Ir1-C5 } & 142.74(13) & \text { C17-Ir1-C5 } & 94.13(15) \\ \text { C4-Ir1-C5 } & 37.88(13) & \text { S1-Ir1-C5 } & 128.13(9) \\ \text { C11-Ir1-C3 } & 96.71(14) & \text { C17-Ir1-C3 } & 133.92(16) \\ \text { C4-Ir1-C3 } & 37.71(13) & \text { S1-Ir1-C3 } & 135.44(10) \\ \text { C5-Ir1-C3 } & 62.31(13) & \text { C11-Ir1-C1 } & 156.45(14) \\ \text { C17-Ir1-C1 } & 122.30(15) & \text { C4-Ir1-C1 } & 62.26(13) \\ \text { S1-Ir1-C1 } & 100.84(9) & \text { C5-Ir1-C1 } & 36.68(13) \\ \text { C3-Ir1-C1 } & 61.43(13) & \text { C11-Ir1-C2 } & 119.40(14) \\ \text { C17-Ir1-C2 } & 156.26(15) & \text { C4-Ir1-C2 } & 62.23(14) \\ \text { S1-Ir1-C2 } & 104.37(11) & \text { C5-Ir1-C2 } & 62.14(14) \\ \text { C3-Ir1-C2 } & 36.10(14) & \text { C1-Ir1-C2 } & 37.46(13) \\ \text { O2-S1-C18 } & 107.2(2) & \text { O2-S1-C19 } & 105.8(2) \\ \text { C18-S1-C19 } & 98.3(2) & \text { O2-S1-Ir1 } & 117.25(13) \\ \text { C18-S1-Ir1 } & 114.99(15) & \text { C19-S1-Ir1 } & 111.29(16) \\ \text { C12-O1-C17 } & 114.0(3) & \text { C5-C1-C2 } & 108.5(3) \\ \text { C5-C1-C6 } & 125.4(3) & \text { C2-C1-C6 } & 125.8(4) \\ \text { C5-C1-Ir1 } & 70.5(2) & \text { C2-C1-Ir1 } & 72.21(18) \\ \text { C6-C1-Ir1 } & 128.2(3) & \text { C3-C2-C1 } & 107.2(3) \\ \text { C3-C2-C7 } & 127.9(4) & \text { C1-C2-C7 } & 124.4(4) \\ \text { C3-C2-Ir1 } & 70.9(2) & \text { C1-C2-Ir1 } & 70.33(18) \\ \text { C7-C2-Ir1 } & 130.4(3) & \text { C2-C3-C4 } & 108.7(3) \\ \text { C2-C3-C8 } & 126.7(4) & \text { C4-C3-C8 } & 124.5(3) \\ \text { C2-C3-Ir1 } & 73.0(2) & \text { C4-C3-Ir1 } & 68.50(19) \\ \text { C8-C3-Ir1 } & 127.5(3) & \text { C5-C4-C3 } & 107.8(3) \\ \text { C5-C4-C9 } & 126.3(3) & \text { C3-C4-C9 } & 125.1(3) \\ \text { C5-C4-Ir1 } & 72.6(2) & \text { C3-C4-Ir1 } & 73.8(2) \\ \text { C9-C4-Ir1 } & 127.3(3) & \text { C1-C5-C4 } & 107.8(3) \\ \text { C1-C5-C10 } & 125.6(3) & \text { C4-C5-C10 } & 126.5(3) \\ \text { C1-C5-Ir1 } & 72.8(2) & \text { C4-C5-Ir1 } & 69.5(2) \\ \text { C10-C5-Ir1 } & 126.6(3) & \text { C1-C6-H6A } & 109.5 \\ \text { C1-C6-H6B } & 109.5 & \text { H6A-C6-H6B } & 109.5 \\ \text { C1-C6-H6C } & 109.5 & \text { H6A-C6-H6C } & 109.5 \\ \text { H6B-C6-H6C } & 109.5 & \text { C2-C7-H7A } & 109.5 \\ \text { C2-C7-H7B } & 109.5 & \text { H7A-C7-H7B } & 109.5 \\ \text { C2-C7-H7C } & 109.5 & \text { H7A-C7-H7C } & 109.5 \\ \text { H7B-C7-H7C } & 109.5 & \text { C3-C8-H8A } & 109.5 \\ \text { C3-C8-H8B } & 109.5 & \text { H8A-C8-H8B } & 109.5 \\ \text { C3-C8-H8C } & 109.5 & \text { H8A-C8-H8C } & 109.5 \\ \text { C1 } 13 & \end{array}$




\begin{tabular}{llll} 
H8B-C8-H8C & 109.5 & C4-C9-H9A & 109.5 \\
C4-C9-H9B & 109.5 & H9A-C9-H9B & 109.5 \\
C4-C9-H9C & 109.5 & H9A-C9-H9C & 109.5 \\
H9B-C9-H9C & 109.5 & C5-C10-H10A & 109.5 \\
C5-C10-H10B & 109.5 & H10A-C10-H10B & 109.5 \\
C5-C10-H10C & 109.5 & H10A-C10-H10C & 109.5 \\
H10B-C10-H10C & 109.5 & C16-C11-C12 & $117.0(3)$ \\
C16-C11-Ir1 & $128.2(3)$ & C12-C11-Ir1 & $114.8(3)$ \\
O1-C12-C13 & $119.1(4)$ & O1-C12-C11 & $118.5(4)$ \\
C13-C12-C11 & $122.3(4)$ & C14-C13-C12 & $118.6(4)$ \\
C14-C13-H13 & 120.7 & C12-C13-H13 & 120.7 \\
C15-C14-C13 & $121.0(4)$ & C15-C14-H14 & 119.5 \\
C13-C14-H14 & 119.5 & C14-C15-C16 & $118.9(4)$ \\
C14-C15-H15 & 120.5 & C16-C15-H15 & 120.5 \\
C11-C16-C15 & $122.0(4)$ & C11-C16-H16 & 119.0 \\
C15-C16-H16 & 119.0 & O1-C17-Ir1 & $113.9(3)$ \\
O1-C17-H17A & 108.8 & Ir1-C17-H17A & 108.8 \\
O1-C17-H17B & 108.8 & Ir1-C17-H17B & 108.8 \\
H17A-C17-H17B & 107.7 & S1-C18-H18A & 109.5 \\
S1-C18-H18B & 109.5 & H18A-C18-H18B & 109.5 \\
S1-C18-H18C & 109.5 & H18A-C18-H18C & 109.5 \\
H18B-C18-H18C & 109.5 & S1-C19-H19A & 109.5 \\
S1-C19-H19B & 109.5 & H19A-C19-H19B & 109.5 \\
S1-C19-H19C & 109.5 & H19A-C19-H19C & 109.5 \\
H19B-C19-H19C & 109.5 & & \\
\hline
\end{tabular}


Torsion angles

Table S14. Torsion angles $\left(^{\circ}\right)$ for 9.

\begin{tabular}{llll} 
C5-C1-C2-C3 & $-0.2(4)$ & C6-C1-C2-C3 & $173.5(3)$ \\
Ir1-C1-C2-C3 & $-61.7(2)$ & C5-C1-C2-C7 & $-172.4(4)$ \\
C6-C1-C2-C7 & $1.3(6)$ & Ir1-C1-C2-C7 & $126.1(4)$ \\
C5-C1-C2-Ir1 & $61.6(3)$ & C6-C1-C2-Ir1 & $-124.7(4)$ \\
C1-C2-C3-C4 & $1.9(4)$ & C7-C2-C3-C4 & $173.7(4)$ \\
Ir1-C2-C3-C4 & $-59.5(2)$ & C1-C2-C3-C8 & $-174.2(3)$ \\
C7-C2-C3-C8 & $-2.4(6)$ & Ir1-C2-C3-C8 & $124.4(4)$ \\
C1-C2-C3-Ir1 & $61.3(2)$ & C7-C2-C3-Ir1 & $-126.8(4)$ \\
C2-C3-C4-C5 & $-2.9(4)$ & C8-C3-C4-C5 & $173.3(3)$ \\
Ir1-C3-C4-C5 & $-65.2(3)$ & C2-C3-C4-C9 & $-173.2(4)$ \\
C8-C3-C4-C9 & $3.1(6)$ & Ir1-C3-C4-C9 & $124.6(4)$ \\
C2-C3-C4-Ir1 & $62.2(2)$ & C8-C3-C4-Ir1 & $-121.5(3)$ \\
C2-C1-C5-C4 & $-1.6(4)$ & C6-C1-C5-C4 & $-175.4(3)$ \\
Ir1-C1-C5-C4 & $61.0(3)$ & C2-C1-C5-C10 & $174.3(3)$ \\
C6-C1-C5-C10 & $0.5(6)$ & Ir1-C1-C5-C10 & $-123.1(4)$ \\
C2-C1-C5-Ir1 & $-62.6(2)$ & C6-C1-C5-Ir1 & $123.6(4)$ \\
C3-C4-C5-C1 & $2.8(4)$ & C9-C4-C5-C1 & $172.9(4)$ \\
Ir1-C4-C5-C1 & $-63.1(3)$ & C3-C4-C5-C10 & $-173.1(4)$ \\
C9-C4-C5-C10 & $-2.9(6)$ & Ir1-C4-C5-C10 & $121.0(4)$ \\
C3-C4-C5-Ir1 & $65.9(2)$ & C9-C4-C5-Ir1 & $-124.0(4)$ \\
C17-O1-C12-C13 & $177.8(3)$ & C17-O1-C12-C11 & $-4.6(5)$ \\
C16-C11-C12-O1 & $-174.2(3)$ & Ir1-C11-C12-O1 & $4.1(4)$ \\
C16-C11-C12-C13 & $3.3(5)$ & Ir1-C11-C12-C13 & $-178.4(3)$ \\
O1-C12-C13-C14 & $175.3(4)$ & C11-C12-C13-C14 & $-2.2(6)$ \\
C12-C13-C14-C15 & $-0.7(6)$ & C13-C14-C15-C16 & $2.4(6)$ \\
C12-C11-C16-C15 & $-1.5(6)$ & Ir1-C11-C16-C15 & $-179.6(3)$ \\
C14-C15-C16-C11 & $-1.2(6)$ & C12-O1-C17-Ir1 & $2.9(4)$ \\
\hline & & &
\end{tabular}


Anisotropic displacement parameters

Table S15. Anisotropic atomic displacement parameters $\left(\AA^{2}\right)$ for 9.

The anisotropic atomic displacement factor exponent takes the form: $-2 \pi^{2}\left[\mathrm{~h}^{2} \mathrm{a}^{* 2} \mathrm{U}_{11}+\ldots\right.$ $+2 \mathrm{~h} \mathrm{k} \mathrm{a}^{*} \mathrm{~b}^{*} \mathrm{U}_{12}$ ]

\begin{tabular}{lllllll} 
& $\mathbf{U}_{11}$ & $\mathbf{U}_{\mathbf{2 2}}$ & $\mathbf{U}_{\mathbf{3 3}}$ & $\mathbf{U}_{\mathbf{2 3}}$ & $\mathbf{U}_{\mathbf{1 3}}$ & $\mathbf{U}_{\mathbf{1 2}}$ \\
$\mathrm{Ir} 1$ & $0.00919(5)$ & $0.00909(4)$ & $0.01728(5)$ & $0.00035(5)$ & $0.00067(5)$ & $0.00012(5)$ \\
$\mathrm{S} 1$ & $0.0121(4)$ & $0.0114(3)$ & $0.0219(4)$ & $-0.0024(3)$ & $-0.0004(3)$ & $0.0013(3)$ \\
$\mathrm{O} 1$ & $0.0158(15)$ & $0.0264(15)$ & $0.0342(15)$ & $0.0089(12)$ & $0.0046(12)$ & $-0.0046(12)$ \\
$\mathrm{O} 2$ & $0.0314(16)$ & $0.0237(13)$ & $0.0341(14)$ & $-0.0130(11)$ & $-0.0114(13)$ & $0.0047(14)$ \\
C1 & $0.0091(13)$ & $0.0161(17)$ & $0.0261(15)$ & $-0.0035(11)$ & $-0.0006(11)$ & $0.0017(11)$ \\
C2 & $0.0123(15)$ & $0.0197(14)$ & $0.0233(14)$ & $-0.0001(16)$ & $0.0051(12)$ & $0.0024(14)$ \\
C3 & $0.0094(14)$ & $0.0176(15)$ & $0.0222(15)$ & $-0.0028(12)$ & $0.0024(11)$ & $0.0011(12)$ \\
C4 & $0.0134(15)$ & $0.0150(15)$ & $0.0229(15)$ & $-0.0042(12)$ & $0.0001(12)$ & $0.0024(12)$ \\
C5 & $0.0141(15)$ & $0.0150(13)$ & $0.0228(14)$ & $-0.0020(11)$ & $-0.0026(14)$ & $0.0050(15)$ \\
C6 & $0.0168(18)$ & $0.0227(18)$ & $0.037(2)$ & $-0.0114(16)$ & $-0.0018(16)$ & $-0.0031(15)$ \\
C7 & $0.022(2)$ & $0.025(2)$ & $0.039(2)$ & $0.0061(17)$ & $0.0093(17)$ & $-0.0042(16)$ \\
C8 & $0.0178(18)$ & $0.034(2)$ & $0.0213(17)$ & $-0.0046(15)$ & $0.0026(13)$ & $0.0017(16)$ \\
C9 & $0.026(2)$ & $0.0134(15)$ & $0.0331(19)$ & $-0.0045(14)$ & $-0.0016(16)$ & $0.0000(15)$ \\
C10 & $0.030(2)$ & $0.0246(18)$ & $0.0240(17)$ & $0.0018(14)$ & $-0.0065(15)$ & $0.0066(16)$ \\
C11 & $0.0087(13)$ & $0.0120(13)$ & $0.0251(14)$ & $-0.0054(12)$ & $0.0008(12)$ & $-0.0007(11)$ \\
C12 & $0.0151(15)$ & $0.0121(13)$ & $0.0331(16)$ & $-0.0022(12)$ & $0.0031(16)$ & $-0.0026(15)$ \\
C13 & $0.0108(17)$ & $0.0216(18)$ & $0.048(2)$ & $-0.0095(18)$ & $0.0051(16)$ & $-0.0040(14)$ \\
C14 & $0.0132(17)$ & $0.032(2)$ & $0.046(2)$ & $-0.0200(19)$ & $-0.0056(16)$ & $0.0025(16)$ \\
C15 & $0.0204(17)$ & $0.033(2)$ & $0.0298(18)$ & $-0.0120(17)$ & $-0.0076(14)$ & $0.0056(16)$ \\
C16 & $0.0134(15)$ & $0.0224(16)$ & $0.0242(15)$ & $-0.0065(14)$ & $-0.0017(11)$ & $0.0008(15)$ \\
C17 & $0.0188(18)$ & $0.0203(17)$ & $0.0303(19)$ & $0.0066(14)$ & $0.0044(14)$ & $-0.0004(15)$ \\
C18 & $0.018(2)$ & $0.0208(19)$ & $0.057(3)$ & $-0.0101(19)$ & $0.0109(18)$ & $0.0027(15)$ \\
C19 & $0.040(3)$ & $0.0190(18)$ & $0.037(2)$ & $0.0077(16)$ & $0.0017(19)$ & $0.0072(19)$ \\
\hline
\end{tabular}


Hydrogen atom coordinates and isotropic atomic displacement parameters

Table S16. Hydrogen atomic coordinates and isotropic atomic displacement parameters $\left(\AA^{2}\right)$ for 9.

\begin{tabular}{lllll} 
& $\mathbf{x} / \mathbf{a}$ & $\mathbf{y} / \mathbf{b}$ & $\mathbf{z} / \mathbf{c}$ & $\mathbf{U}(\mathbf{e q})$ \\
H6A & 0.9188 & 0.5811 & 1.0337 & 0.038 \\
H6B & 0.9643 & 0.6450 & 0.9482 & 0.038 \\
H6C & 1.0714 & 0.5596 & 0.9849 & 0.038 \\
H7A & 1.0157 & 0.5922 & 0.7474 & 0.043 \\
H7B & 0.9114 & 0.6630 & 0.8055 & 0.043 \\
H7C & 0.8492 & 0.6103 & 0.7178 & 0.043 \\
H8A & 0.7860 & 0.4548 & 0.6603 & 0.037 \\
H8B & 0.6820 & 0.3658 & 0.6935 & 0.037 \\
H8C & 0.8530 & 0.3458 & 0.6774 & 0.037 \\
H9A & 0.8427 & 0.2064 & 0.8470 & 0.036 \\
H9B & 0.6833 & 0.2375 & 0.8121 & 0.036 \\
H9C & 0.7123 & 0.2246 & 0.9162 & 0.036 \\
H10A & 0.9787 & 0.3442 & 1.0423 & 0.039 \\
H10B & 0.8158 & 0.2996 & 1.0392 & 0.039 \\
H10C & 0.8441 & 0.4089 & 1.0793 & 0.039 \\
H13 & 0.1427 & 0.3547 & 0.8561 & 0.032 \\
H14 & 0.1062 & 0.4014 & 0.7080 & 0.037 \\
H15 & 0.2862 & 0.4898 & 0.6313 & 0.033 \\
H16 & 0.5116 & 0.5224 & 0.7004 & 0.024 \\
H17A & 0.5604 & 0.3535 & 1.0085 & 0.028 \\
H17B & 0.5029 & 0.4615 & 1.0381 & 0.028 \\
H18A & 0.3569 & 0.6017 & 1.0098 & 0.048 \\
H18B & 0.3211 & 0.6060 & 0.9059 & 0.048 \\
H18C & 0.3453 & 0.7074 & 0.9604 & 0.048 \\
H19A & 0.5311 & 0.7811 & 0.8557 & 0.048 \\
H19B & 0.5049 & 0.6882 & 0.7906 & 0.048 \\
H19C & 0.6684 & 0.7221 & 0.8151 & 0.048 \\
\hline
\end{tabular}




\section{Crystal Structure Report for 2}

Crystal description

A yellow block-like specimen of $\mathrm{C}_{14} \mathrm{H}_{24} \mathrm{Cl}_{2} \mathrm{IrNO}$, approximate dimensions $0.090 \mathrm{~mm} \mathrm{x}$ $0.130 \mathrm{~mm} \times 0.250 \mathrm{~mm}$, was used for the X-ray crystallographic analysis. Instrument description The X-ray intensity data were measured.

Data collection The total exposure time was 7.16 hours. Integration The frames were integrated with the Bruker SAINT software package using a narrow-frame algorithm. The integration of the data using a monoclinic unit cell yielded a total of 32594 reflections to a maximum $\theta$ angle of $33.25^{\circ}(0.65 \AA$ resolution), of which 6023 were independent (average redundancy 5.412, completeness $=99.9 \%, \mathrm{R}_{\text {int }}=2.81 \%, \mathrm{R}_{\text {sig }}=2.06 \%$ ) and 5480 $(90.98 \%)$ were greater than $2 \sigma\left(\mathrm{F}^{2}\right)$. Unit cell The final cell constants of $\underline{a}=15.4301(5) \AA$, $\underline{\mathrm{b}}=7.6155(3) \AA, \underline{\mathrm{c}}=14.5741(5) \AA, \beta=114.0590(10)^{\circ}$, volume $=1563.79(10) \AA^{3}$, are based upon the refinement of the XYZ-centroids of 9901 reflections above $20 \sigma(\mathrm{I})$ with $5.604^{\circ}<2 \theta<66.47^{\circ}$. Scaling Data were corrected for absorption effects using the multiscan method (SADABS). The ratio of minimum to maximum apparent transmission was 0.644 .

Structure solution The structure was solved and refined using the Bruker SHELXTL Software Package, using the space group P 121 /c 1 , with $Z=4$ for the formula unit, $\mathrm{C}_{14} \mathrm{H}_{24} \mathrm{Cl}_{2} \mathrm{IrNO}$. Structure refinement The final anisotropic full-matrix least-squares refinement on $\mathrm{F}^{2}$ with 181 variables converged at $\mathrm{R} 1=1.70 \%$, for the observed data and $\mathrm{wR} 2=4.24 \%$ for all data. The goodness-of-fit was 1.026 . The largest peak in the final difference electron density synthesis was $1.999 \mathrm{e}^{-} / \AA^{3}$ and the largest hole was $-0.894 \mathrm{e}^{-}$ $/ \AA^{3}$ with an RMS deviation of $0.132 \mathrm{e}^{-} / \AA^{3}$. On the basis of the final model, the calculated density was $2.062 \mathrm{~g} / \mathrm{cm}^{3}$ and $F(000), 936 \mathrm{e}^{-}$. Structure Packing Sample and crystal data 
Table S17. Sample and crystal data for 2.

Identification code $\quad \mathrm{rds} 038$

Chemical formula $\quad \mathrm{C}_{14} \mathrm{H}_{24} \mathrm{Cl}_{2} \mathrm{IrNO}$

Formula weight $\quad 485.44 \mathrm{~g} / \mathrm{mol}$

Temperature $\quad 110(2) \mathrm{K}$

Wavelength $\quad 0.71073 \AA$

Crystal size

Crystal habit

Crystal system

Space group

$0.090 \times 0.130 \times 0.250 \mathrm{~mm}$

Unit cell dimensions yellow block

monoclinic

P $121 / \mathrm{c} 1$

$a=15.4301(5) \AA \quad \alpha=90^{\circ}$

$\mathrm{b}=7.6155(3) \AA \quad \beta=114.0590(10)^{\circ}$

$\mathrm{c}=14.5741(5) \AA \gamma=90^{\circ}$

Volume $\quad 1563.79(10) \AA^{3}$

Z 4

Density (calculated) $\quad 2.062 \mathrm{~g} / \mathrm{cm}^{3}$

Absorption coefficient $8.872 \mathrm{~mm}^{-1}$

$\mathrm{F}(000)$

936 
Data collection and structure refinement

Table S18. Data collection and structure refinement for 2.

Theta range for data collection

2.80 to $33.25^{\circ}$

Index ranges

$-20<=\mathrm{h}<=23,-11<=\mathrm{k}<=11,-22<=\mathrm{l}<=22$

Reflections collected

32594

Independent reflections

$6023[\mathrm{R}(\mathrm{int})=0.0281]$

Coverage of independent reflections

Absorption correction

$99.9 \%$

Structure solution technique

multi-scan

direct methods

Structure solution program

Refinement method

Refinement program

SHELXS-97 (Sheldrick, 2008)

Function minimized

Full-matrix least-squares on $\mathrm{F}^{2}$

SHELXL-97 (Sheldrick, 2008)

$\Sigma \mathrm{w}\left(\mathrm{F}_{\mathrm{o}}^{2}-\mathrm{F}_{\mathrm{c}}^{2}\right)^{2}$

Data / restraints / parameters

$6023 / 0 / 181$

Goodness-of-fit on $\mathrm{F}^{2}$

1.026

$\Delta / \sigma_{\max }$

0.005

Final $\mathrm{R}$ indices

5480 data; $\mathrm{I}>2 \sigma(\mathrm{I}) \quad \mathrm{R} 1=0.0170, \mathrm{wR} 2=0.0409$

all data $\quad \mathrm{R} 1=0.0204, \mathrm{wR} 2=0.0424$

Weighting scheme

$\mathrm{w}=1 /\left[\sigma^{2}\left(\mathrm{~F}_{\mathrm{o}}{ }^{2}\right)+(0.0231 \mathrm{P})^{2}+0.6764 \mathrm{P}\right]$

Largest diff. peak and hole

where $\mathrm{P}=\left(\mathrm{F}_{\mathrm{o}}{ }^{2}+2 \mathrm{~F}_{\mathrm{c}}{ }^{2}\right) / 3$

R.M.S. deviation from mean 1.999 and $-0.894 \mathrm{e}^{-3}$

$0.132 \mathrm{e}^{-3}$ 
Atomic coordinates and equivalent isotropic displacement parameters

Table S19. Atomic coordinates and equivalent isotropic atomic displacement parameters $\left(\AA^{2}\right)$ for 2.

$\mathrm{U}(\mathrm{eq})$ is defined as one third of the trace of the orthogonalized $\mathrm{U}_{\mathrm{ij}}$ tensor.

\begin{tabular}{lllll}
\hline & $\mathbf{x} / \mathbf{a}$ & $\mathbf{y} / \mathbf{b}$ & $\mathbf{z} / \mathbf{c}$ & $\mathbf{U}(\mathbf{e q})$ \\
$\mathrm{Ir} 1$ & $0.717563(4)$ & $0.807297(8)$ & $0.814972(5)$ & $0.00927(2)$ \\
$\mathrm{C} 11$ & $0.66224(3)$ & $0.56351(6)$ & $0.70045(3)$ & $0.01596(8)$ \\
$\mathrm{C} 12$ & $0.76446(3)$ & $0.59754(6)$ & $0.95148(3)$ & $0.01613(8)$ \\
O1 & $0.03060(11)$ & $0.69972(18)$ & $0.79735(13)$ & $0.0209(3)$ \\
N1 & $0.84911(11)$ & $0.7233(2)$ & $0.80838(12)$ & $0.0115(3)$ \\
C1 & $0.71029(13)$ & $0.0360(2)$ & $0.89768(13)$ & $0.0119(3)$ \\
C5 & $0.73309(13)$ & $0.0885(2)$ & $0.81560(13)$ & $0.0124(3)$ \\
C4 & $0.65739(13)$ & $0.0330(2)$ & $0.72259(13)$ & $0.0124(3)$ \\
C9 & $0.64912(15)$ & $0.0708(3)$ & $0.61872(15)$ & $0.0187(4)$ \\
C3 & $0.58545(13)$ & $0.9499(2)$ & $0.74880(14)$ & $0.0130(3)$ \\
C2 & $0.61803(12)$ & $0.9495(2)$ & $0.85572(13)$ & $0.0121(3)$ \\
C7 & $0.56726(14)$ & $0.8789(3)$ & $0.91579(15)$ & $0.0172(3)$ \\
C8 & $0.49276(14)$ & $0.8775(3)$ & $0.67552(15)$ & $0.0193(4)$ \\
C10 & $0.81883(15)$ & $0.1927(2)$ & $0.82652(17)$ & $0.0182(4)$ \\
C6 & $0.76632(14)$ & $0.0784(3)$ & $0.00564(14)$ & $0.0172(3)$ \\
C14 & $0.93973(14)$ & $0.7474(3)$ & $0.89795(15)$ & $0.0166(3)$ \\
C13 & $0.01819(14)$ & $0.6439(3)$ & $0.88462(16)$ & $0.0188(4)$ \\
C12 & $0.94436(15)$ & $0.6757(3)$ & $0.70986(16)$ & $0.0181(4)$ \\
C11 & $0.86268(15)$ & $0.7771(2)$ & $0.71735(15)$ & $0.0157(3)$ \\
\hline
\end{tabular}


Bond lengths

Table S20. Bond lengths ( $\AA$ ) for 2.

\begin{tabular}{llll} 
Ir1-C1 & $2.1466(17)$ & Ir1-C4 & $2.1470(17)$ \\
Ir1-C2 & $2.1513(17)$ & Ir1-C5 & $2.1547(18)$ \\
Ir1-C3 & $2.1590(17)$ & Ir1-N1 & $2.1673(16)$ \\
Ir1-C11 & $2.4083(4)$ & Ir1-C12 & $2.4206(4)$ \\
O1-C13 & $1.426(3)$ & O1-C12 & $1.431(3)$ \\
N1-C11 & $1.483(2)$ & N1-C14 & $1.485(2)$ \\
N1-H1 & $0.86(3)$ & C1-C5 & $1.435(2)$ \\
C1-C2 & $1.457(3)$ & C1-C6 & $1.489(3)$ \\
C5-C4 & $1.446(3)$ & C5-C10 & $1.494(3)$ \\
C4-C3 & $1.458(3)$ & C4-C9 & $1.495(3)$ \\
C9-H9A & 0.98 & C9-H9B & 0.98 \\
C9-H9C & 0.98 & C3-C2 & $1.429(3)$ \\
C3-C8 & $1.498(3)$ & C2-C7 & $1.493(3)$ \\
C7-H7A & 0.98 & C7-H7B & 0.98 \\
C7-H7C & 0.98 & C8-H8A & 0.98 \\
C8-H8B & 0.98 & C8-H8C & 0.98 \\
C10-H10A & 0.98 & C10-H10B & 0.98 \\
C10-H10C & 0.98 & C6-H6A & 0.98 \\
C6-H6B & 0.98 & C6-H6C & 0.98 \\
C14-C13 & $1.522(3)$ & C14-H14A & 0.99 \\
C14-H14B & 0.99 & C13-H13A & 0.99 \\
C13-H13B & 0.99 & C12-C11 & $1.520(3)$ \\
C12-H12A & 0.99 & C12-H12B & 0.99 \\
C11-H11A & 0.99 & C11-H11B & 0.99 \\
\hline
\end{tabular}


Bond angles

Table S21. Bond angles $\left(^{\circ}\right)$ for 2.

$\begin{array}{llll}\text { C1-Ir1-C4 } & 66.08(7) & \text { C1-Ir1-C2 } & 39.64(7) \\ \text { C4-Ir1-C2 } & 66.12(7) & \text { C1-Ir1-C5 } & 38.96(7) \\ \text { C4-Ir1-C5 } & 39.27(7) & \text { C2-Ir1-C5 } & 65.73(7) \\ \text { C1-Ir1-C3 } & 65.60(7) & \text { C4-Ir1-C3 } & 39.57(7) \\ \text { C2-Ir1-C3 } & 38.72(7) & \text { C5-Ir1-C3 } & 65.49(7) \\ \text { C1-Ir1-N1 } & 121.91(6) & \text { C4-Ir1-N1 } & 112.45(6) \\ \text { C2-Ir1-N1 } & 161.38(6) & \text { C5-Ir1-N1 } & 100.89(6) \\ \text { C3-Ir1-N1 } & 149.24(6) & \text { C1-Ir1-C11 } & 158.38(5) \\ \text { C4-Ir1-C11 } & 104.01(5) & \text { C2-Ir1-C11 } & 119.23(5) \\ \text { C5-Ir1-C11 } & 140.95(5) & \text { C3-Ir1-C11 } & 94.29(5) \\ \text { N1-Ir1-C11 } & 79.37(4) & \text { C1-Ir1-C12 } & 98.07(5) \\ \text { C4-Ir1-C12 } & 162.11(5) & \text { C2-Ir1-C12 } & 96.50(5) \\ \text { C5-Ir1-C12 } & 131.07(5) & \text { C3-Ir1-C12 } & 127.62(5) \\ \text { N1-Ir1-C12 } & 82.54(4) & \text { C11-Ir1-C12 } & 87.915(16) \\ \text { C13-O1-C12 } & 110.02(15) & \text { C11-N1-C14 } & 109.07(15) \\ \text { C11-N1-Ir1 } & 115.76(11) & \text { C14-N1-Ir1 } & 119.20(12) \\ \text { C11-N1-H1 } & 104.8(18) & \text { C14-N1-H1 } & 107.8(18) \\ \text { Ir1-N1-H1 } & 98.3(17) & \text { C5-C1-C2 } & 107.81(15) \\ \text { C5-C1-C6 } & 125.89(17) & \text { C2-C1-C6 } & 126.03(16) \\ \text { C5-C1-Ir1 } & 70.82(10) & \text { C2-C1-Ir1 } & 70.35(10) \\ \text { C6-C1-Ir1 } & 128.94(13) & \text { C1-C5-C4 } & 108.73(16) \\ \text { C1-C5-C10 } & 124.52(17) & \text { C4-C5-C10 } & 126.66(17) \\ \text { C1-C5-Ir1 } & 70.22(10) & \text { C4-C5-Ir1 } & 70.08(10) \\ \text { C10-C5-Ir1 } & 128.32(13) & \text { C5-C4-C3 } & 106.97(15) \\ \text { C5-C4-C9 } & 126.60(17) & \text { C3-C4-C9 } & 126.12(17) \\ \text { C5-C4-Ir1 } & 70.65(10) & \text { C3-C4-Ir1 } & 70.66(10) \\ \text { C9-C4-Ir1 } & 128.75(13) & \text { C4-C9-H9A } & 109.5 \\ \text { C4-C9-H9B } & 109.5 & \text { H9A-C9-H9B } & 109.5 \\ \text { C4-C9-H9C } & 109.5 & \text { H9A-C9-H9C } & 109.5 \\ \text { H9B-C9-H9C } & 109.5 & \text { C2-C3-C4 } & 108.63(16) \\ \text { C2-C3-C8 } & 125.87(17) & \text { C4-C3-C8 } & 125.50(17) \\ \text { C2-C3-Ir1 } & 70.35(10) & \text { C4-C3-Ir1 } & 69.77(10) \\ \text { C8-C3-Ir1 } & 125.92(13) & \text { C3-C2-C1 } & 107.82(15) \\ \text { C3-C2-C7 } & 127.20(17) & \text { C1-C2-C7 } & 124.97(16) \\ \text { C3-C2-Ir1 } & 70.93(10) & \text { C1-C2-Ir1 } & 70.01(9) \\ \text { C7-C2-Ir1 } & 125.79(12) & \text { C2-C7-H7A } & 109.5 \\ \text { C2-C7-H7B } & 109.5 & \text { H7A-C7-H7B } & 109.5 \\ \text { C2-C7-H7C } & 109.5 & \text { H7A-C7-H7C } & 109.5 \\ \text { H7B-C7-H7C } & 109.5 & \text { C3-C8-H8A } & 109.5 \\ \text { C3-C8-H8B } & 109.5 & \text { H8A-C8-H8B } & 109.5 \\ \text { C3-C8-H8C } & 109.5 & \text { H8A-C8-H8C } & 109.5\end{array}$




\begin{tabular}{llll} 
H8B-C8-H8C & 109.5 & C5-C10-H10A & 109.5 \\
C5-C10-H10B & 109.5 & H10A-C10-H10B & 109.5 \\
C5-C10-H10C & 109.5 & H10A-C10-H10C & 109.5 \\
H10B-C10-H10C & 109.5 & C1-C6-H6A & 109.5 \\
C1-C6-H6B & 109.5 & H6A-C6-H6B & 109.5 \\
C1-C6-H6C & 109.5 & H6A-C6-H6C & 109.5 \\
H6B-C6-H6C & 109.5 & N1-C14-C13 & $109.69(16)$ \\
N1-C14-H14A & 109.7 & C13-C14-H14A & 109.7 \\
N1-C14-H14B & 109.7 & C13-C14-H14B & 109.7 \\
H14A-C14-H14B & 108.2 & O1-C13-C14 & $111.28(17)$ \\
O1-C13-H13A & 109.4 & C14-C13-H13A & 109.4 \\
O1-C13-H13B & 109.4 & C14-C13-H13B & 109.4 \\
H13A-C13-H13B & 108.0 & O1-C12-C11 & $111.60(17)$ \\
O1-C12-H12A & 109.3 & C11-C12-H12A & 109.3 \\
O1-C12-H12B & 109.3 & C11-C12-H12B & 109.3 \\
H12A-C12-H12B & 108.0 & N1-C11-C12 & $110.35(16)$ \\
N1-C11-H11A & 109.6 & C12-C11-H11A & 109.6 \\
N1-C11-H11B & 109.6 & C12-C11-H11B & 109.6 \\
H11A-C11-H11B & 108.1 & & \\
\hline
\end{tabular}


Torsion angles

Table S22. Torsion angles $\left({ }^{\circ}\right)$ for 2.

\begin{tabular}{|c|c|c|c|}
\hline C1-Ir1-N1-C11 & $-98.07(13)$ & C4-Ir1-N1-C11 & $-23.12(14)$ \\
\hline C2-Ir1-N1-C11 & $-104.7(2)$ & C5-Ir1-N1-C11 & $-62.46(13)$ \\
\hline C3-Ir1-N1-C11 & $-2.6(2)$ & C11-Ir1-N1-C11 & $77.77(12)$ \\
\hline Cl2-Ir1-N1-C11 & $167.04(13)$ & C1-Ir1-N1-C14 & $35.19(16)$ \\
\hline C4-Ir1-N1-C14 & $110.14(14)$ & C2-Ir1-N1-C14 & $28.5(3)$ \\
\hline C5-Ir1-N1-C14 & $70.81(14)$ & C3-Ir1-N1-C14 & $130.63(15)$ \\
\hline C11-Ir1-N1-C14 & $-148.97(14)$ & $\mathrm{C} 12-\mathrm{Ir} 1-\mathrm{N} 1-\mathrm{C} 14$ & $-59.70(13)$ \\
\hline C4-Ir1-C1-C5 & $-37.04(10)$ & C2-Ir1-C1-C5 & $-117.90(15)$ \\
\hline C3-Ir1-C1-C5 & $-80.59(11)$ & N1-Ir1-C1-C5 & $65.43(12)$ \\
\hline C11-Ir1-C1-C5 & $-103.42(14)$ & $\mathrm{Cl} 2-\mathrm{Ir} 1-\mathrm{C} 1-\mathrm{C} 5$ & $151.63(9)$ \\
\hline C4-Ir1-C1-C2 & $80.87(11)$ & C5-Ir1-C1-C2 & $117.90(15)$ \\
\hline C3-Ir1-C1-C2 & $37.32(10)$ & N1-Ir1-C1-C2 & $-176.67(9)$ \\
\hline C11-Ir1-C1-C2 & $14.49(19)$ & $\mathrm{Cl} 2-\mathrm{Ir} 1-\mathrm{C} 1-\mathrm{C} 2$ & $-90.47(9)$ \\
\hline C4-Ir1-C1-C6 & $-158.17(19)$ & C2-Ir1-C1-C6 & $121.0(2)$ \\
\hline C5-Ir1-C1-C6 & $-121.1(2)$ & C3-Ir1-C1-C6 & 158.28(19) \\
\hline N1-Ir1-C1-C6 & $-55.70(19)$ & Cl1-Ir1-C1-C6 & $135.45(14)$ \\
\hline $\mathrm{C} 12-\mathrm{Ir} 1-\mathrm{C} 1-\mathrm{C} 6$ & $30.50(17)$ & $\mathrm{C} 2-\mathrm{C} 1-\mathrm{C} 5-\mathrm{C} 4$ & $-1.24(19)$ \\
\hline C6-C1-C5-C4 & $-175.56(17)$ & Ir1-C1-C5-C4 & $59.71(12)$ \\
\hline $\mathrm{C} 2-\mathrm{C} 1-\mathrm{C} 5-\mathrm{C} 10$ & $175.52(16)$ & C6-C1-C5-C10 & $1.2(3)$ \\
\hline Ir1-C1-C5-C10 & $-123.53(17)$ & C2-C1-C5-Ir1 & $-60.95(12)$ \\
\hline C6-C1-C5-Ir1 & $124.73(18)$ & C4-Ir1-C5-C1 & $119.57(15)$ \\
\hline C2-Ir1-C5-C1 & $38.20(10)$ & C3-Ir1-C5-C1 & $80.90(11)$ \\
\hline N1-Ir1-C5-C1 & $-128.17(10)$ & Cl1-Ir1-C5-C1 & $145.33(9)$ \\
\hline $\mathrm{Cl} 2-\operatorname{Ir} 1-\mathrm{C} 5-\mathrm{C} 1$ & $-38.61(12)$ & C1-Ir1-C5-C4 & $-119.57(15)$ \\
\hline C2-Ir1-C5-C4 & $-81.36(11)$ & C3-Ir1-C5-C4 & $-38.67(10)$ \\
\hline N1-Ir1-C5-C4 & $112.27(10)$ & Cl1-Ir1-C5-C4 & $25.76(14)$ \\
\hline Cl2-Ir1-C5-C4 & $-158.18(8)$ & C1-Ir1-C5-C10 & $118.9(2)$ \\
\hline C4-Ir1-C5-C10 & $-121.5(2)$ & C2-Ir1-C5-C10 & $157.10(19)$ \\
\hline C3-Ir1-C5-C10 & $-160.20(19)$ & N1-Ir1-C5-C10 & $-9.27(18)$ \\
\hline C11-Ir1-C5-C10 & $-95.77(17)$ & C12-Ir1-C5-C10 & $80.29(18)$ \\
\hline $\mathrm{C} 1-\mathrm{C} 5-\mathrm{C} 4-\mathrm{C} 3$ & $1.90(19)$ & C10-C5-C4-C3 & $-174.77(17)$ \\
\hline Ir1-C5-C4-C3 & $61.69(11)$ & C1-C5-C4-C9 & $175.77(17)$ \\
\hline C10-C5-C4-C9 & $-0.9(3)$ & Ir1-C5-C4-C9 & $-124.44(18)$ \\
\hline C1-C5-C4-Ir1 & $-59.80(12)$ & C10-C5-C4-Ir1 & $123.54(18)$ \\
\hline C1-Ir1-C4-C5 & $36.75(10)$ & C2-Ir1-C4-C5 & $80.29(11)$ \\
\hline C3-Ir1-C4-C5 & $116.82(15)$ & N1-Ir1-C4-C5 & $-79.50(11)$ \\
\hline C11-Ir1-C4-C5 & $-163.61(9)$ & $\mathrm{Cl} 2-\mathrm{Ir} 1-\mathrm{C} 4-\mathrm{C} 5$ & $65.8(2)$ \\
\hline C1-Ir1-C4-C3 & $-80.06(11)$ & C2-Ir1-C4-C3 & $-36.52(10)$ \\
\hline C5-Ir1-C4-C3 & $-116.82(15)$ & N1-Ir1-C4-C3 & $163.68(10)$ \\
\hline C11-Ir1-C4-C3 & $79.57(10)$ & $\mathrm{Cl} 2-\mathrm{Ir} 1-\mathrm{C} 4-\mathrm{C} 3$ & $-51.0(2)$ \\
\hline C1-Ir1-C4-C9 & $158.65(19)$ & C2-Ir1-C4-C9 & $-157.81(19)$ \\
\hline
\end{tabular}




\begin{tabular}{llll} 
C5-Ir1-C4-C9 & $121.9(2)$ & C3-Ir1-C4-C9 & $-121.3(2)$ \\
N1-Ir1-C4-C9 & $42.39(19)$ & C11-Ir1-C4-C9 & $-41.71(18)$ \\
C12-Ir1-C4-C9 & $-172.30(12)$ & C5-C4-C3-C2 & $-1.85(19)$ \\
C9-C4-C3-C2 & $-175.76(16)$ & Ir1-C4-C3-C2 & $59.83(12)$ \\
C5-C4-C3-C8 & $177.98(17)$ & C9-C4-C3-C8 & $4.1(3)$ \\
Ir1-C4-C3-C8 & $-120.33(18)$ & C5-C4-C3-Ir1 & $-61.69(12)$ \\
C9-C4-C3-Ir1 & $124.40(18)$ & C1-Ir1-C3-C2 & $-38.19(10)$ \\
C4-Ir1-C3-C2 & $-119.55(15)$ & C5-Ir1-C3-C2 & $-81.17(11)$ \\
N1-Ir1-C3-C2 & $-150.07(12)$ & C11-Ir1-C3-C2 & $133.57(10)$ \\
C12-Ir1-C3-C2 & $42.90(12)$ & C1-Ir1-C3-C4 & $81.36(11)$ \\
C2-Ir1-C3-C4 & $119.55(15)$ & C5-Ir1-C3-C4 & $38.38(10)$ \\
N1-Ir1-C3-C4 & $-30.52(17)$ & C11-Ir1-C3-C4 & $-106.88(10)$ \\
C12-Ir1-C3-C4 & $162.45(8)$ & C1-Ir1-C3-C8 & $-158.82(18)$ \\
C4-Ir1-C3-C8 & $119.8(2)$ & C2-Ir1-C3-C8 & $-120.6(2)$ \\
C5-Ir1-C3-C8 & $158.19(18)$ & N1-Ir1-C3-C8 & $89.3(2)$ \\
C11-Ir1-C3-C8 & $12.94(16)$ & C12-Ir1-C3-C8 & $-77.74(17)$ \\
C4-C3-C2-C1 & $1.11(19)$ & C8-C3-C2-C1 & $-178.72(17)$ \\
Ir1-C3-C2-C1 & $60.59(12)$ & C4-C3-C2-C7 & $179.57(17)$ \\
C8-C3-C2-C7 & $-0.3(3)$ & Ir1-C3-C2-C7 & $-120.95(18)$ \\
C4-C3-C2-Ir1 & $-59.48(12)$ & C8-C3-C2-Ir1 & $120.69(18)$ \\
C5-C1-C2-C3 & $0.07(19)$ & C6-C1-C2-C3 & $174.38(17)$ \\
Ir1-C1-C2-C3 & $-61.17(12)$ & C5-C1-C2-C7 & $-178.43(16)$ \\
C6-C1-C2-C7 & $-4.1(3)$ & Ir1-C1-C2-C7 & $120.32(17)$ \\
C5-C1-C2-Ir1 & $61.25(12)$ & C6-C1-C2-Ir1 & $-124.44(18)$ \\
C1-Ir1-C2-C3 & $118.05(15)$ & C4-Ir1-C2-C3 & $37.30(10)$ \\
C5-Ir1-C2-C3 & $80.49(11)$ & N1-Ir1-C2-C3 & $126.94(19)$ \\
C11-Ir1-C2-C3 & $-55.88(11)$ & C12-Ir1-C2-C3 & $-147.13(10)$ \\
C4-Ir1-C2-C1 & $-80.75(11)$ & C5-Ir1-C2-C1 & $-37.56(10)$ \\
C3-Ir1-C2-C1 & $-118.05(15)$ & N1-Ir1-C2-C1 & $8.9(2)$ \\
C11-Ir1-C2-C1 & $-173.94(8)$ & C12-Ir1-C2-C1 & $94.81(9)$ \\
C1-Ir1-C2-C7 & $-119.3(2)$ & C4-Ir1-C2-C7 & $159.94(18)$ \\
C5-Ir1-C2-C7 & $-156.87(18)$ & C3-Ir1-C2-C7 & $122.6(2)$ \\
N1-Ir1-C2-C7 & $-110.4(2)$ & C11-Ir1-C2-C7 & $66.76(17)$ \\
C12-Ir1-C2-C7 & $-24.49(16)$ & C11-N1-C14-C13 & $-56.5(2)$ \\
Ir1-N1-C14-C13 & $167.45(13)$ & C12-O1-C13-C14 & $-59.6(2)$ \\
N1-C14-C13-O1 & $59.2(2)$ & C13-O1-C12-C11 & $58.6(2)$ \\
C14-N1-C11-C12 & $55.7(2)$ & Ir1-N1-C11-C12 & $-166.54(13)$ \\
O1-C12-C11-N1 & $-57.3(2)$ & & \\
\hline & & & \\
\hline
\end{tabular}


Anisotropic displacement parameters

Table S23. Anisotropic atomic displacement parameters $\left(\AA^{2}\right)$ for $\mathbf{2}$.

The anisotropic atomic displacement factor exponent takes the form: $-2 \pi^{2}\left[\mathrm{~h}^{2} \mathrm{a}^{* 2} \mathrm{U}_{11}+\ldots\right.$ $+2 \mathrm{~h} \mathrm{k} \mathrm{a}^{*} \mathrm{~b}^{*} \mathrm{U}_{12}$ ]

\begin{tabular}{|c|c|c|c|c|c|c|}
\hline & & & & $\mathbf{U}_{23}$ & & $\mathbf{U}_{12}$ \\
\hline Ir1 & $0.00938(3)$ & $0.00944(3)$ & $0.00976(3)$ & $0.00066(2)$ & $0.00468(2)$ & $0.00075(2)$ \\
\hline $\mathrm{Cl1}$ & $\begin{array}{l}0.01450(19 \\
)\end{array}$ & $\begin{array}{l}0.01561(18 \\
)\end{array}$ & $\begin{array}{l}0.01663(19 \\
)\end{array}$ & $0.00448(15$ & $\begin{array}{l}0.00516(15 \\
)\end{array}$ & $0.00140(15$ \\
\hline $\mathrm{Cl} 2$ & $0.0200(2)$ & $0.01532(18$ & $0.01520(18$ & $0.00524(15$ & $0.00939(16$ & $0.00435(15$ \\
\hline $\mathrm{O} 1$ & $0.0135(7)$ & $0.0230(7)$ & $0.0290(8)$ & $-0.0014(6)$ & $0.0115(6)$ & $-0.0001(5)$ \\
\hline N1 & $0.0114(7)$ & $0.0108(6)$ & $0.0127(6)$ & $0.0004(5)$ & $0.0053(5)$ & $0.0008(5)$ \\
\hline $\mathrm{C} 1$ & $0.0134(8)$ & $0.0105(7)$ & $0.0132(7)$ & $-0.0003(6)$ & $0.0068(6)$ & $0.0016(6)$ \\
\hline $\mathrm{C} 5$ & $0.0142(8)$ & $0.0098(7)$ & $0.0159(8)$ & $0.0008(6)$ & $0.0088(6)$ & $0.0019(6)$ \\
\hline $\mathrm{C} 4$ & $0.0146(8)$ & $0.0108(7)$ & $0.0121(7)$ & $0.0032(6)$ & $0.0057(6)$ & $0.0041(6)$ \\
\hline C9 & $0.0250(10)$ & $0.0193(8)$ & $0.0151(8)$ & $0.0049(7)$ & $0.0113(7)$ & $0.0061(7)$ \\
\hline C3 & $0.0129(8)$ & $0.0119(7)$ & $0.0138(7)$ & $0.0008(6)$ & $0.0051(6)$ & $0.0035(6)$ \\
\hline $\mathrm{C} 2$ & $0.0112(7)$ & $0.0126(7)$ & $0.0136(7)$ & $0.0013(6)$ & $0.0063(6)$ & $0.0033(6)$ \\
\hline $\mathrm{C} 7$ & $0.0162(9)$ & $0.0199(9)$ & $0.0194(9)$ & $0.0042(7)$ & $0.0112(7)$ & $0.0026(7)$ \\
\hline $\mathrm{C} 8$ & $0.0144(8)$ & $0.0209(9)$ & $0.0183(9)$ & $0.0003(7)$ & $0.0023(7)$ & $0.0021(7)$ \\
\hline $\begin{array}{l}\mathrm{C} 1 \\
0\end{array}$ & $0.0203(9)$ & $0.0132(8)$ & $0.0265(10)$ & $0.0002(7)$ & $0.0152(8)$ & $-0.0011(7)$ \\
\hline C6 & $0.0188(9)$ & $0.0184(8)$ & $0.0126(7)$ & $-0.0039(7)$ & $0.0047(7)$ & $-0.0008(7)$ \\
\hline $\begin{array}{l}\mathrm{C} 1 \\
4\end{array}$ & $0.0120(8)$ & $0.0202(8)$ & $0.0159(8)$ & $-0.0024(7)$ & $0.0041(7)$ & $0.0007(7)$ \\
\hline $\begin{array}{l}\mathrm{C} 1 \\
3\end{array}$ & $0.0116(8)$ & $0.0215(8)$ & $0.0221(9)$ & $-0.0003(7)$ & $0.0056(7)$ & $0.0024(7)$ \\
\hline $\begin{array}{l}\mathrm{C} 1 \\
2\end{array}$ & $0.0175(9)$ & $0.0193(8)$ & $0.0217(9)$ & $-0.0004(7)$ & $0.0122(8)$ & $0.0011(7)$ \\
\hline C1 & $0.0182(9)$ & $0.0153(8)$ & $0.0174(8)$ & $0.0029(6)$ & $0.0113(7)$ & $0.0027(7)$ \\
\hline
\end{tabular}


Hydrogen atom coordinates and isotropic atomic displacement parameters

Table S24. Hydrogen atomic coordinates and isotropic atomic displacement parameters $\left(\AA^{2}\right)$ for 2.

\begin{tabular}{lllll} 
& $\mathbf{x} / \mathbf{a}$ & $\mathbf{y} / \mathbf{b}$ & $\mathbf{z} / \mathbf{c}$ & $\mathbf{U}(\mathbf{e q})$ \\
H9A & 0.6269 & 1.1916 & 0.6004 & 0.028 \\
H9B & 0.6037 & 0.9891 & 0.5713 & 0.028 \\
H9C & 0.7113 & 1.0564 & 0.6164 & 0.028 \\
H7A & 0.5348 & 0.9750 & 0.9336 & 0.026 \\
H7B & 0.6131 & 0.8240 & 0.9772 & 0.026 \\
H7C & 0.5205 & 0.7913 & 0.8760 & 0.026 \\
H8A & 0.4723 & 0.7821 & 0.7073 & 0.029 \\
H8B & 0.5005 & 0.8323 & 0.6163 & 0.029 \\
H8C & 0.4448 & 0.9707 & 0.6550 & 0.029 \\
H10A & 0.8080 & 1.3169 & 0.8360 & 0.027 \\
H10B & 0.8308 & 1.1792 & 0.7658 & 0.027 \\
H10C & 0.8739 & 1.1502 & 0.8849 & 0.027 \\
H6A & 0.8331 & 1.0481 & 1.0243 & 0.026 \\
H6B & 0.7417 & 1.0109 & 1.0471 & 0.026 \\
H6C & 0.7610 & 1.2042 & 1.0166 & 0.026 \\
H14A & 0.9324 & 0.7061 & 0.9588 & 0.02 \\
H14B & 0.9566 & 0.8735 & 0.9068 & 0.02 \\
H13A & 1.0784 & 0.6601 & 0.9446 & 0.023 \\
H13B & 1.0022 & 0.5174 & 0.8787 & 0.023 \\
H12A & 0.9283 & 0.5492 & 0.7014 & 0.022 \\
H12B & 0.9537 & 0.7156 & 0.6499 & 0.022 \\
H11A & 0.8762 & 0.9045 & 0.7204 & 0.019 \\
H11B & 0.8037 & 0.7545 & 0.6569 & 0.019 \\
H1 & $0.8374(19)$ & $0.612(4)$ & $0.801(2)$ & $0.024(7)$ \\
\hline
\end{tabular}




\section{Crystal Structure Report for 12}

Crystal description

A yellow plate-like specimen of $\mathrm{C}_{22} \mathrm{H}_{39} \mathrm{IrO}_{5} \mathrm{~S}$, approximate dimensions $0.144 \mathrm{~mm} \times 0.314$ $\mathrm{mm} \times 0.336 \mathrm{~mm}$, was used for the X-ray crystallographic analysis. Instrument description The X-ray intensity data were measured.

Data collection The total exposure time was 3.13 hours. Integration The frames were integrated with the Bruker SAINT software package using a narrow-frame algorithm. The integration of the data using a triclinic unit cell yielded a total of 105563 reflections to a maximum $\theta$ angle of $36.38^{\circ}$ ( $0.60 \AA$ resolution), of which 12020 were independent (average redundancy 8.782, completeness $=99.9 \%, \mathrm{R}_{\text {int }}=3.18 \%, \mathrm{R}_{\text {sig }}=1.83 \%$ ) and $11385(94.72 \%)$ were greater than $2 \sigma\left(\mathrm{F}^{2}\right)$. Unit cell The final cell constants of $\underline{\mathrm{a}}=$ 8.8769(3) $\AA, \underline{b}=9.5555(3) \AA, \underline{c}=14.9869(4) \AA, \alpha=85.124(2)^{\circ}, \beta=84.523(2)^{\circ}, \gamma=$ $78.209(2)^{\circ}$, volume $=1235.93(7) \AA^{3}$, are based upon the refinement of the XYZcentroids of 239 reflections above $20 \sigma(\mathrm{I})$ with $6.935^{\circ}<2 \theta<68.99^{\circ}$. Scaling Data were corrected for absorption effects using the numerical method (SADABS). The ratio of minimum to maximum apparent transmission was 0.476 . The calculated minimum and maximum transmission coefficients (based on crystal size) are 0.2590 and 0.5040 .

Structure solution Structure refinement The final anisotropic full-matrix least-squares refinement on $\mathrm{F}^{2}$ with 306 variables converged at $\mathrm{R} 1=1.45 \%$, for the observed data and $\mathrm{wR} 2=3.19 \%$ for all data. The goodness-of-fit was 1.062 . The largest peak in the final difference electron density synthesis was $0.980 \mathrm{e}^{-} / \AA^{3}$ and the largest hole was $-0.715 \mathrm{e}^{-}$ $/ \AA^{3}$ with an RMS deviation of $0.093 \mathrm{e}^{-} / \AA^{3}$. On the basis of the final model, the calculated density was $1.633 \mathrm{~g} / \mathrm{cm}^{3}$ and $\mathrm{F}(000), 608 \mathrm{e}^{-}$. Structure Packing Sample and crystal data 
Table S25. Sample and crystal data for 12.

Identification code $\quad \operatorname{rds} 252$

Chemical formula

Formula weight

$\mathrm{C}_{22} \mathrm{H}_{39} \mathrm{IrO}_{5} \mathrm{~S}$

Temperature

Wavelength

607.82

Crystal size

Crystal habit

Crystal system

Space group

$110(2) \mathrm{K}$

$0.71073 \AA$

$0.144 \times 0.314 \times 0.336 \mathrm{~mm}$

yellow plate

triclinic

Unit cell dimensions

$\mathrm{P}-1$

$\mathrm{a}=8.8769(3) \AA \quad \alpha=85.124(2)^{\circ}$

$\mathrm{b}=9.5555(3) \AA \quad \beta=84.523(2)^{\circ}$

$\mathrm{c}=14.9869(4) \AA \quad \gamma=78.209(2)^{\circ}$

Volume 1235.93(7) $\AA^{3}$

Z

2

Density (calculated) $\quad 1.633 \mathrm{~g} / \mathrm{cm}^{3}$

Absorption coefficient $5.514 \mathrm{~mm}^{-1}$

$\mathrm{F}(000)$

608 
Data collection and structure refinement

Table S26. Data collection and structure refinement for $\mathbf{1 2 .}$

Theta range for data collection

Index ranges

Reflections collected

Independent reflections

Coverage of independent reflections

Absorption correction

Max. and min. transmission

Refinement method

Refinement program

Function minimized

Data / restraints / parameters

Goodness-of-fit on $\mathrm{F}^{2}$

$\Delta / \sigma_{\max }$

Final $\mathrm{R}$ indices

Weighting scheme

Largest diff. peak and hole

R.M.S. deviation from mean
2.18 to $36.38^{\circ}$

$-14<=\mathrm{h}<=14,-15<=\mathrm{k}<=15,-24<=\mathrm{l}<=24$

105563

$12020[\mathrm{R}(\mathrm{int})=0.0318]$

$99.9 \%$

numerical

0.5040 and 0.2590

Full-matrix least-squares on $\mathrm{F}^{2}$

SHELXL-2013 (Sheldrick, 2013)

$\Sigma \mathrm{w}\left(\mathrm{F}_{\mathrm{o}}{ }^{2}-\mathrm{F}_{\mathrm{c}}{ }^{2}\right)^{2}$

12020 / 27 / 306

1.062

0.007

11385 data; $\mathrm{I}>2 \sigma(\mathrm{I}) \quad \mathrm{R} 1=0.0145, \mathrm{wR} 2=0.0315$

all data $\quad \mathrm{R} 1=0.0165, \mathrm{wR} 2=0.0319$

$\mathrm{W}=1 /\left[\sigma^{2}\left(\mathrm{~F}_{\mathrm{o}}^{2}\right)+(0.0139 \mathrm{P})^{2}+0.4109 \mathrm{P}\right]$

where $\mathrm{P}=\left(\mathrm{F}_{\mathrm{o}}{ }^{2}+2 \mathrm{~F}_{\mathrm{c}}{ }^{2}\right) / 3$

0.980 and $-0.715 \mathrm{e}^{-3}$

$0.093 \mathrm{e}^{-3}$ 
Atomic coordinates and equivalent isotropic displacement parameters

Table S27. Atomic coordinates and equivalent isotropic atomic displacement parameters $\left(\AA^{2}\right)$ for 12.

$\mathrm{U}(\mathrm{eq})$ is defined as one third of the trace of the orthogonalized $\mathrm{U}_{\mathrm{ij}}$ tensor.

\begin{tabular}{lllll}
\hline & $\mathbf{x} / \mathbf{a}$ & $\mathbf{y} / \mathbf{b}$ & $\mathbf{z} / \mathbf{c}$ & $\mathbf{U}(\mathbf{e q})$ \\
Ir1 & $0.75566(2)$ & $0.52163(2)$ & $0.72757(2)$ & $0.00872(10)$ \\
S1 & $0.57667(3)$ & $0.41233(3)$ & $0.81274(2)$ & $0.01269(4)$ \\
O1 & $0.52442(11)$ & $0.30187(10)$ & $0.76711(6)$ & $0.02085(18)$ \\
O2 & $0.60808(9)$ & $0.71955(9)$ & $0.73190(5)$ & $0.01309(14)$ \\
O3 & $0.42409(10)$ & $0.66940(10)$ & $0.65365(6)$ & $0.01722(16)$ \\
O4 & $0.84554(9)$ & $0.60815(10)$ & $0.83068(6)$ & $0.01539(15)$ \\
O5 & $0.65023(11)$ & $0.65011(11)$ & $0.93745(6)$ & $0.02211(19)$ \\
C1 & $0.87878(13)$ & $0.32933(13)$ & $0.66541(8)$ & $0.01521(19)$ \\
C2 & $0.98573(13)$ & $0.41445(14)$ & $0.68316(8)$ & $0.0178(2)$ \\
C3 & $0.94824(13)$ & $0.55168(14)$ & $0.63259(8)$ & $0.0167(2)$ \\
C4 & $0.81862(13)$ & $0.54856(13)$ & $0.58334(7)$ & $0.01478(19)$ \\
C5 & $0.77137(12)$ & $0.41392(12)$ & $0.60457(7)$ & $0.01268(18)$ \\
C6 & $0.87995(16)$ & $0.17652(14)$ & $0.69722(10)$ & $0.0236(2)$ \\
C7 & $0.11538(15)$ & $0.37343(19)$ & $0.74315(11)$ & $0.0298(3)$ \\
C8 & $0.03402(18)$ & $0.67132(18)$ & $0.63114(11)$ & $0.0311(3)$ \\
C9 & $0.74403(17)$ & $0.66681(15)$ & $0.52100(8)$ & $0.0230(2)$ \\
C10 & $0.64416(14)$ & $0.36465(14)$ & $0.56521(8)$ & $0.0181(2)$ \\
C11 & $0.65287(16)$ & $0.32657(15)$ & $0.91354(8)$ & $0.0203(2)$ \\
C12 & $0.40808(13)$ & $0.52946(14)$ & $0.85550(8)$ & $0.0170(2)$ \\
C13 & $0.48237(12)$ & $0.75328(12)$ & $0.69037(7)$ & $0.01200(17)$ \\
C14 & $0.40950(13)$ & $0.91364(13)$ & $0.69262(8)$ & $0.0162(2)$ \\
C15 & $0.52840(18)$ & $0.00208(16)$ & $0.65423(13)$ & $0.0324(3)$ \\
C16 & $0.3624(3)$ & $0.94692(19)$ & $0.79049(11)$ & $0.0429(5)$ \\
C17 & $0.27065(17)$ & $0.95067(16)$ & $0.63707(13)$ & $0.0320(3)$ \\
C18 & $0.77362(13)$ & $0.67053(13)$ & $0.90027(7)$ & $0.01347(18)$ \\
C19 & $0.85759(14)$ & $0.77788(14)$ & $0.93580(8)$ & $0.0169(2)$ \\
C20 & $0.0172(3)$ & $0.7058(3)$ & $0.9568(2)$ & $0.0442(8)$ \\
C21 & $0.7644(3)$ & $0.8456(3)$ & $0.01787(16)$ & $0.0404(7)$ \\
C22 & $0.8639(3)$ & $0.8988(2)$ & $0.86174(15)$ & $0.0325(5)$ \\
C20A & $0.0270(10)$ & $0.7628(13)$ & $0.8964(6)$ & $0.064(3)$ \\
C21A & $0.8658(9)$ & $0.7435(8)$ & $0.0394(4)$ & $0.0352(16)$ \\
C22A & $0.7677(13)$ & $0.9223(8)$ & $0.9229(8)$ & $0.065(3)$ \\
\hline & & & &
\end{tabular}


Bond lengths

Table S28. Bond lengths ( $\AA$ ) for 12.

$\begin{array}{llll}\text { Ir1-O2 } & 2.0710(8) & \text { Ir1-O4 } & 2.1048(8) \\ \text { Ir1-C2 } & 2.1597(11) & \text { Ir1-C5 } & 2.1659(10) \\ \text { Ir1-C3 } & 2.1674(11) & \text { Ir1-C1 } & 2.1713(11) \\ \text { Ir1-C4 } & 2.1873(11) & \text { Ir1-S1 } & 2.3041(3) \\ \text { S1-O1 } & 1.4767(9) & \text { S1-C12 } & 1.7769(12) \\ \text { S1-C11 } & 1.7803(13) & \text { O2-C13 } & 1.2993(13) \\ \text { O3-C13 } & 1.2295(14) & \text { O4-C18 } & 1.2938(13) \\ \text { O5-C18 } & 1.2251(14) & \text { C1-C2 } & 1.4263(17) \\ \text { C1-C5 } & 1.4558(16) & \text { C1-C6 } & 1.4953(18) \\ \text { C2-C3 } & 1.4510(19) & \text { C2-C7 } & 1.4965(18) \\ \text { C3-C4 } & 1.4316(17) & \text { C3-C8 } & 1.4952(18) \\ \text { C4-C5 } & 1.4345(16) & \text { C4-C9 } & 1.4904(18) \\ \text { C5-C10 } & 1.4955(16) & \text { C6-H6A } & 0.98 \\ \text { C6-H6B } & 0.98 & \text { C6-H6C } & 0.98 \\ \text { C7-H7A } & 0.98 & \text { C7-H7B } & 0.98 \\ \text { C7-H7C } & 0.98 & \text { C8-H8A } & 0.98 \\ \text { C8-H8B } & 0.98 & \text { C8-H8C } & 0.98 \\ \text { C9-H9A } & 0.98 & \text { C9-H9B } & 0.98 \\ \text { C9-H9C } & 0.98 & \text { C10-H10A } & 0.98 \\ \text { C10-H10B } & 0.98 & \text { C10-H10C } & 0.98 \\ \text { C11-H11A } & 0.98 & \text { C11-H11B } & 0.98 \\ \text { C11-H11C } & 0.98 & \text { C12-H12A } & 0.98 \\ \text { C12-H12B } & 0.98 & \text { C12-H12C } & 0.98 \\ \text { C13-C14 } & 1.5385(16) & \text { C14-C17 } & 1.5183(19) \\ \text { C14-C15 } & 1.5249(19) & \text { C14-C16 } & 1.528(2) \\ \text { C15-H15A } & 0.98 & \text { C15-H15B } & 0.98 \\ \text { C15-H15C } & 0.98 & \text { C16-H16A } & 0.98 \\ \text { C16-H16B } & 0.98 & \text { C16-H16C } & 0.98 \\ \text { C17-H17A } & 0.98 & \text { C17-H17B } & 0.98 \\ \text { C17-H17C } & 0.98 & \text { C18-C19 } & 1.5403(16) \\ \text { C19-C22A } & 1.454(8) & \text { C19-C20 } & 1.494(3) \\ \text { C19-C21 } & 1.529(2) & \text { C19-C22 } & 1.538(2) \\ \text { C19-C20A } & 1.545(7) & \text { C19-C21A } & 1.565(6) \\ \text { C20-H20A } & 0.98 & \text { C20-H20B } & 0.98 \\ \text { C20-H20C } & 0.98 & \text { C21-H21A } & 0.98 \\ \text { C21-H21B } & 0.98 & \text { C21-H21C } & 0.98 \\ \text { C22-H22A } & 0.98 & \text { C22-H22B } & 0.98 \\ \text { C22-H22C } & 0.98 & \text { C20A-H20D } & 0.98 \\ \text { C20A-H20E } & 0.98 & \text { C20A-H20F } & 0.98 \\ \text { C21A-H21D } & 0.98 & \text { C21A-H21E } & 0.98 \\ \text { C21A-H21F } & 0.98 & \text { C22A-H22D } & 0.98\end{array}$




\begin{tabular}{llll}
$\mathrm{C} 22 \mathrm{~A}-\mathrm{H} 22 \mathrm{E}$ & 0.98 & $\mathrm{C} 22 \mathrm{~A}-\mathrm{H} 22 \mathrm{~F}$ & 0.98 \\
\hline
\end{tabular}

Bond angles

Table S29. Bond angles $\left(^{\circ}\right)$ for 12.

$\begin{array}{llll}\text { O2-Ir1-O4 } & 79.82(3) & \text { O2-Ir1-C2 } & 143.79(4) \\ \text { O4-Ir1-C2 } & 89.98(4) & \text { O2-Ir1-C5 } & 116.34(4) \\ \text { O4-Ir1-C5 } & 154.56(4) & \text { C2-Ir1-C5 } & 65.26(4) \\ \text { O2-Ir1-C3 } & 106.09(4) & \text { O4-Ir1-C3 } & 92.27(4) \\ \text { C2-Ir1-C3 } & 39.19(5) & \text { C5-Ir1-C3 } & 65.12(4) \\ \text { O2-Ir1-C1 } & 155.43(4) & \text { O4-Ir1-C1 } & 121.95(4) \\ \text { C2-Ir1-C1 } & 38.45(4) & \text { C5-Ir1-C1 } & 39.22(4) \\ \text { C3-Ir1-C1 } & 65.01(5) & \text { O2-Ir1-C4 } & 93.94(4) \\ \text { O4-Ir1-C4 } & 126.61(4) & \text { C2-Ir1-C4 } & 64.64(5) \\ \text { C5-Ir1-C4 } & 38.48(4) & \text { C3-Ir1-C4 } & 38.38(4) \\ \text { C1-Ir1-C4 } & 64.55(5) & \text { O2-Ir1-S1 } & 91.38(2) \\ \text { O4-Ir1-S1 } & 99.28(2) & \text { C2-Ir1-S1 } & 124.67(4) \\ \text { C5-Ir1-S1 } & 99.77(3) & \text { C3-Ir1-S1 } & 160.57(3) \\ \text { C1-Ir1-S1 } & 95.57(3) & \text { C4-Ir1-S1 } & 134.03(3) \\ \text { O1-S1-C12 } & 106.88(6) & \text { O1-S1-C11 } & 106.55(6) \\ \text { C12-S1-C11 } & 101.51(6) & \text { O1-S1-Ir1 } & 114.33(4) \\ \text { C12-S1-Ir1 } & 115.59(4) & \text { C11-S1-Ir1 } & 110.87(4) \\ \text { C13-O2-Ir1 } & 123.53(7) & \text { C18-O4-Ir1 } & 129.21(7) \\ \text { C2-C1-C5 } & 108.06(11) & \text { C2-C1-C6 } & 127.19(11) \\ \text { C5-C1-C6 } & 124.63(11) & \text { C2-C1-Ir1 } & 70.33(6) \\ \text { C5-C1-Ir1 } & 70.19(6) & \text { C6-C1-Ir1 } & 128.26(9) \\ \text { C1-C2-C3 } & 108.25(10) & \text { C1-C2-C7 } & 127.26(13) \\ \text { C3-C2-C7 } & 124.49(12) & \text { C1-C2-Ir1 } & 71.21(6) \\ \text { C3-C2-Ir1 } & 70.69(6) & \text { C7-C2-Ir1 } & 123.74(9) \\ \text { C4-C3-C2 } & 107.46(10) & \text { C4-C3-C8 } & 127.08(13) \\ \text { C2-C3-C8 } & 125.44(12) & \text { C4-C3-Ir1 } & 71.56(6) \\ \text { C2-C3-Ir1 } & 70.12(6) & \text { C8-C3-Ir1 } & 125.01(8) \\ \text { C3-C4-C5 } & 108.91(11) & \text { C3-C4-C9 } & 125.43(11) \\ \text { C5-C4-C9 } & 125.65(11) & \text { C3-C4-Ir1 } & 70.06(6) \\ \text { C5-C4-Ir1 } & 69.96(6) & \text { C9-C4-Ir1 } & 125.50(8) \\ \text { C4-C5-C1 } & 107.26(10) & \text { C4-C5-C10 } & 126.30(11) \\ \text { C1-C5-C10 } & 126.24(11) & \text { C4-C5-Ir1 } & 71.57(6) \\ \text { C1-C5-Ir1 } & 70.59(6) & \text { C10-C5-Ir1 } & 127.12(8) \\ \text { C1-C6-H6A } & 109.5 & \text { C1-C6-H6B } & 109.5 \\ \text { H6A-C6-H6B } & 109.5 & \text { C1-C6-H6C } & 109.5 \\ \text { H6A-C6-H6C } & 109.5 & \text { H6B-C6-H6C } & 109.5 \\ \text { C2-C7-H7A } & 109.5 & \text { C2-C7-H7B } & 109.5 \\ \text { H7A-C7-H7B } & 109.5 & \text { C2-C7-H7C } & 109.5 \\ \text { H7A-C7-H7C } & 109.5 & \text { H7B-C7-H7C } & 109.5 \\ \text { C3-C8-H8A } & 109.5 & \text { C3-C8-H8B } & 109.5 \\ \text { H8A-C8-H8B } & 109.5 & \text { C3-C8-H8C } & 109.5\end{array}$




\begin{tabular}{|c|c|c|c|}
\hline H8A-C8-H8C & 109.5 & H8B-C8-H8C & 109.5 \\
\hline C4-C9-H9A & 109.5 & C4-C9-H9B & 109.5 \\
\hline H9A-C9-H9B & 109.5 & C4-C9-H9C & 109.5 \\
\hline H9A-C9-H9C & 109.5 & H9B-C9-H9C & 109.5 \\
\hline C5-C10-H10A & 109.5 & C5-C10-H10B & 109.5 \\
\hline H10A-C10-H10B & 109.5 & C5-C10-H10C & 109.5 \\
\hline H10A-C10-H10C & 109.5 & H10B-C10-H10C & 109.5 \\
\hline S1-C11-H11A & 109.5 & S1-C11-H11B & 109.5 \\
\hline H11A-C11-H11B & 109.5 & S1-C11-H11C & 109.5 \\
\hline H11A-C11-H11C & 109.5 & H11B-C11-H11C & 109.5 \\
\hline S1-C12-H12A & 109.5 & $\mathrm{~S} 1-\mathrm{C} 12-\mathrm{H} 12 \mathrm{~B}$ & 109.5 \\
\hline H12A-C12-H12B & 109.5 & $\mathrm{~S} 1-\mathrm{C} 12-\mathrm{H} 12 \mathrm{C}$ & 109.5 \\
\hline H12A-C12-H12C & 109.5 & H12B-C12-H12C & 109.5 \\
\hline O3-C13-O2 & $125.46(11)$ & O3-C13-C14 & $122.27(10)$ \\
\hline $\mathrm{O} 2-\mathrm{C} 13-\mathrm{C} 14$ & $112.27(9)$ & C17-C14-C15 & $108.97(12)$ \\
\hline C17-C14-C16 & $110.74(13)$ & C15-C14-C16 & $109.02(14)$ \\
\hline C17-C14-C13 & $110.52(10)$ & C15-C14-C13 & $109.37(10)$ \\
\hline C16-C14-C13 & $108.20(11)$ & C14-C15-H15A & 109.5 \\
\hline C14-C15-H15B & 109.5 & H15A-C15-H15B & 109.5 \\
\hline C14-C15-H15C & 109.5 & H15A-C15-H15C & 109.5 \\
\hline H15B-C15-H15C & 109.5 & C14-C16-H16A & 109.5 \\
\hline C14-C16-H16B & 109.5 & H16A-C16-H16B & 109.5 \\
\hline C14-C16-H16C & 109.5 & H16A-C16-H16C & 109.5 \\
\hline H16B-C16-H16C & 109.5 & C14-C17-H17A & 109.5 \\
\hline C14-C17-H17B & 109.5 & H17A-C17-H17B & 109.5 \\
\hline C14-C17-H17C & 109.5 & H17A-C17-H17C & 109.5 \\
\hline H17B-C17-H17C & 109.5 & O5-C18-O4 & $125.67(11)$ \\
\hline O5-C18-C19 & $120.42(10)$ & O4-C18-C19 & $113.91(10)$ \\
\hline C20-C19-C21 & $111.56(19)$ & C20-C19-C22 & $110.17(18)$ \\
\hline C21-C19-C22 & $107.26(17)$ & C22A-C19-C18 & $109.6(3)$ \\
\hline C20-C19-C18 & $110.36(13)$ & C21-C19-C18 & $110.09(12)$ \\
\hline C22-C19-C18 & $107.26(11)$ & C22A-C19-C20A & $113.3(7)$ \\
\hline C18-C19-C20A & $112.9(3)$ & C22A-C19-C21A & $107.1(5)$ \\
\hline C18-C19-C21A & $108.0(2)$ & C20A-C19-C21A & $105.5(5)$ \\
\hline C19-C20-H20A & 109.5 & C19-C20-H20B & 109.5 \\
\hline H20A-C20-H20B & 109.5 & C19-C20-H20C & 109.5 \\
\hline H20A-C20-H20C & 109.5 & H20B-C20-H20C & 109.5 \\
\hline C19-C21-H21A & 109.5 & C19-C21-H21B & 109.5 \\
\hline H21A-C21-H21B & 109.5 & C19-C21-H21C & 109.5 \\
\hline $\mathrm{H} 21 \mathrm{~A}-\mathrm{C} 21-\mathrm{H} 21 \mathrm{C}$ & 109.5 & $\mathrm{H} 21 \mathrm{~B}-\mathrm{C} 21-\mathrm{H} 21 \mathrm{C}$ & 109.5 \\
\hline C19-C22-H22A & 109.5 & C19-C22-H22B & 109.5 \\
\hline $\mathrm{H} 22 \mathrm{~A}-\mathrm{C} 22-\mathrm{H} 22 \mathrm{~B}$ & 109.5 & C19-C22-H22C & 109.5 \\
\hline $\mathrm{H} 22 \mathrm{~A}-\mathrm{C} 22-\mathrm{H} 22 \mathrm{C}$ & 109.5 & $\mathrm{H} 22 \mathrm{~B}-\mathrm{C} 22-\mathrm{H} 22 \mathrm{C}$ & 109.5 \\
\hline C19-C20A-H20D & 109.5 & C19-C20A-H20E & 109.5 \\
\hline H20D-C20A-H20E & 109.5 & C19-C20A-H20F & 109.5 \\
\hline H20D-C20A-H20F & 109.5 & H20E-C20A-H20F & 109.5 \\
\hline
\end{tabular}




\begin{tabular}{llll} 
C19-C21A-H21D & 109.5 & C19-C21A-H21E & 109.5 \\
H21D-C21A-H21E & 109.5 & C19-C21A-H21F & 109.5 \\
H21D-C21A-H21F & 109.5 & H21E-C21A-H21F & 109.5 \\
C19-C22A-H22D & 109.5 & C19-C22A-H22E & 109.5 \\
H22D-C22A-H22E & 109.5 & C19-C22A-H22F & 109.5 \\
H22D-C22A-H22F & 109.5 & H22E-C22A-H22F & 109.5 \\
\hline
\end{tabular}


Torsion angles

Table S30. Torsion angles $\left({ }^{\circ}\right)$ for 12.

\begin{tabular}{llll} 
C5-C1-C2-C3 & $-1.00(12)$ & C6-C1-C2-C3 & $175.02(11)$ \\
Ir1-C1-C2-C3 & $-61.32(8)$ & C5-C1-C2-C7 & $178.90(12)$ \\
C6-C1-C2-C7 & $-5.1(2)$ & Ir1-C1-C2-C7 & $118.58(13)$ \\
C5-C1-C2-Ir1 & $60.32(7)$ & C6-C1-C2-Ir1 & $-123.65(12)$ \\
C1-C2-C3-C4 & $-0.55(12)$ & C7-C2-C3-C4 & $179.54(11)$ \\
Ir1-C2-C3-C4 & $-62.20(7)$ & C1-C2-C3-C8 & $-178.92(11)$ \\
C7-C2-C3-C8 & $1.18(19)$ & Ir1-C2-C3-C8 & $119.43(12)$ \\
C1-C2-C3-Ir1 & $61.65(8)$ & C7-C2-C3-Ir1 & $-118.25(12)$ \\
C2-C3-C4-C5 & $1.92(12)$ & C8-C3-C4-C5 & $-179.75(11)$ \\
Ir1-C3-C4-C5 & $-59.35(7)$ & C2-C3-C4-C9 & $-178.72(10)$ \\
C8-C3-C4-C9 & $-0.39(18)$ & Ir1-C3-C4-C9 & $120.01(11)$ \\
C2-C3-C4-Ir1 & $61.27(7)$ & C8-C3-C4-Ir1 & $-120.40(12)$ \\
C3-C4-C5-C1 & $-2.52(12)$ & C9-C4-C5-C1 & $178.12(10)$ \\
Ir1-C4-C5-C1 & $-61.94(7)$ & C3-C4-C5-C10 & $-177.60(10)$ \\
C9-C4-C5-C10 & $3.05(18)$ & Ir1-C4-C5-C10 & $122.99(11)$ \\
C3-C4-C5-Ir1 & $59.42(7)$ & C9-C4-C5-Ir1 & $-119.94(11)$ \\
C2-C1-C5-C4 & $2.16(12)$ & C6-C1-C5-C4 & $-173.99(11)$ \\
Ir1-C1-C5-C4 & $62.58(7)$ & C2-C1-C5-C10 & $177.24(10)$ \\
C6-C1-C5-C10 & $1.09(18)$ & Ir1-C1-C5-C10 & $-122.34(11)$ \\
C2-C1-C5-Ir1 & $-60.41(8)$ & C6-C1-C5-Ir1 & $123.44(12)$ \\
Ir1-O2-C13-O3 & $10.02(16)$ & Ir1-O2-C13-C14 & $-170.74(7)$ \\
O3-C13-C14-C17 & $-5.34(17)$ & O2-C13-C14-C17 & $175.40(11)$ \\
O3-C13-C14-C15 & $-125.30(13)$ & O2-C13-C14-C15 & $55.44(14)$ \\
O3-C13-C14-C16 & $116.07(15)$ & O2-C13-C14-C16 & $-63.19(15)$ \\
Ir1-O4-C18-O5 & $26.94(18)$ & Ir1-O4-C18-C19 & $-153.26(8)$ \\
O5-C18-C19-C22A & $-66.8(6)$ & O4-C18-C19-C22A & $113.4(6)$ \\
O5-C18-C19-C20 & $123.54(19)$ & O4-C18-C19-C20 & $-56.3(2)$ \\
O5-C18-C19-C21 & $0.0(2)$ & O4-C18-C19-C21 & $-179.86(16)$ \\
O5-C18-C19-C22 & $-116.43(16)$ & O4-C18-C19-C22 & $63.76(16)$ \\
O5-C18-C19-C20A & $165.9(6)$ & O4-C18-C19-C20A & $-14.0(6)$ \\
O5-C18-C19-C21A & $49.6(4)$ & O4-C18-C19-C21A & $-130.3(3)$ \\
\hline & & &
\end{tabular}


Anisotropic displacement parameters

Table S31. Anisotropic atomic displacement parameters $\left(\AA^{2}\right)$ for $\mathbf{1 2}$.

The anisotropic atomic displacement factor exponent takes the form: $-2 \pi^{2}\left[\mathrm{~h}^{2} \mathrm{a}^{* 2} \mathrm{U}_{11}+\ldots\right.$ $\left.+2 \mathrm{~h} \mathrm{k} \mathrm{a}^{*} \mathrm{~b}^{*} \mathrm{U}_{12}\right]$

$\begin{array}{llllll}\mathbf{U}_{11} & \mathrm{U}_{22} & \mathrm{U}_{33} & \mathrm{U}_{23} & \mathrm{U}_{13} & \mathbf{U}_{12}\end{array}$

\begin{tabular}{|c|c|c|c|c|c|c|}
\hline Ir1 & $0.00719(2)$ & $0.01096(2)$ & $0.00837(2)$ & $0.00279(10$ & $0.00034(10$ & $0.00191(10$ \\
\hline \multirow[t]{2}{*}{1} & $0.01325(10$ & $0.01459(12$ & $0.01144(10$ & - & \multirow{2}{*}{$0.00073(8)$} & 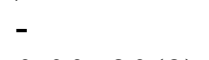 \\
\hline & & ) & ) & $0.00199(9)$ & & $0.00590(9)$ \\
\hline 1 & $0.0249(4)$ & $0.0212(4)$ & $0.0208(4)$ & $-0.0070(3)$ & $0.0017(3)$ & $-0.0140(4)$ \\
\hline 2 & $0.0118(3)$ & $0.0129(4)$ & $0.0144(3)$ & $-0.0036(3)$ & $-0.0023(3)$ & $-0.0003(3)$ \\
\hline 3 & $0.0166(4)$ & $0.0155(4)$ & $0.0208(4)$ & $-0.0040(3)$ & $-0.0057(3)$ & $-0.0028(3)$ \\
\hline 4 & $0.0120(3)$ & $0.0221(4)$ & $0.0135(3)$ & $-0.0083(3)$ & $-0.0020(3)$ & $-0.0033(3)$ \\
\hline 5 & $0.0220(4)$ & $0.0306(5)$ & $0.0171(4)$ & $-0.0081(4)$ & $0.0050(3)$ & $-0.0135(4)$ \\
\hline 1 & $0.0123(4)$ & $0.0165(5)$ & $0.0163(5)$ & $-0.0069(4)$ & $-0.0007(3)$ & $0.0005(4)$ \\
\hline 2 & $0.0090(4)$ & $0.0238(6)$ & $0.0207(5)$ & $-0.0107(4)$ & $-0.0006(4)$ & $-0.0001(4)$ \\
\hline 3 & $0.0115(4)$ & $0.0233(6)$ & $0.0170(5)$ & $-0.0093(4)$ & $0.0065(4)$ & $-0.0079(4)$ \\
\hline $\mathrm{C} 4$ & $0.0158(4)$ & $0.0186(5)$ & $0.0103(4)$ & $-0.0046(4)$ & $0.0041(3)$ & $-0.0049(4)$ \\
\hline $\mathrm{C} 5$ & $0.0120(4)$ & $0.0158(5)$ & $0.0105(4)$ & $-0.0051(3)$ & $0.0004(3)$ & $-0.0024(4)$ \\
\hline C6 & $0.0244(6)$ & $0.0143(5)$ & $0.0300(6)$ & $-0.0040(5)$ & $-0.0041(5)$ & $0.0027(4)$ \\
\hline $\mathrm{C} 7$ & $0.0140(5)$ & $0.0397(8)$ & $0.0356(7)$ & $-0.0175(6)$ & $-0.0102(5)$ & $0.0046(5)$ \\
\hline $\mathrm{C} 8$ & $0.0261(6)$ & $0.0367(8)$ & $0.0364(8)$ & $-0.0178(6)$ & $0.0151(6)$ & $-0.0215(6)$ \\
\hline C9 & $0.0304(6)$ & $0.0238(6)$ & $0.0129(5)$ & $0.0016(4)$ & $0.0035(4)$ & $-0.0041(5)$ \\
\hline $\mathrm{C} 10$ & $0.0180(5)$ & $0.0231(6)$ & $0.0159(5)$ & $-0.0078(4)$ & $-0.0030(4)$ & $-0.0068(4)$ \\
\hline $\mathrm{C} 11$ & $0.0238(6)$ & $0.0213(6)$ & $0.0153(5)$ & $0.0024(4)$ & $-0.0011(4)$ & $-0.0053(5)$ \\
\hline C12 & $0.0129(4)$ & $0.0224(6)$ & $0.0160(5)$ & $-0.0021(4)$ & $0.0024(4)$ & $-0.0055(4)$ \\
\hline C13 & $0.0110(4)$ & $0.0125(4)$ & $0.0121(4)$ & $-0.0019(3)$ & $0.0003(3)$ & $-0.0015(3)$ \\
\hline C14 & $0.0152(5)$ & $0.0115(5)$ & $0.0209(5)$ & $-0.0023(4)$ & $-0.0014(4)$ & $-0.0001(4)$ \\
\hline C15 & $0.0249(6)$ & $0.0156(6)$ & $0.0573(10)$ & $0.0009(6)$ & $-0.0024(6)$ & $-0.0072(5)$ \\
\hline $\mathrm{C} 16$ & $0.0611(12)$ & $0.0281(8)$ & $0.0270(7)$ & $-0.0081(6)$ & $0.0064(7)$ & $0.0183(8)$ \\
\hline $\mathrm{C} 17$ & $0.0234(6)$ & $0.0168(6)$ & $0.0562(10)$ & $0.0007(6)$ & $-0.0187(6)$ & $0.0010(5)$ \\
\hline C18 & $0.0157(4)$ & $0.0158(5)$ & $0.0100(4)$ & $-0.0022(3)$ & $-0.0022(3)$ & $-0.0046(4)$ \\
\hline C19 & $0.0216(5)$ & $0.0188(5)$ & $0.0130(4)$ & $-0.0038(4)$ & $-0.0024(4)$ & $-0.0088(4)$ \\
\hline $\mathrm{C}$ & $0.0324(11)$ & $0.0286(11)$ & $0.078(2)$ & $0.0092(12)$ & $0.0344(13)$ & $-0.0053(9)$ \\
\hline & $0.0552(15)$ & $0.0460(15)$ & $0.0288(10)$ & $\begin{array}{l}- \\
0.0240(10)\end{array}$ & $0.0120(10)$ & $\begin{array}{l}- \\
0.0289(12)\end{array}$ \\
\hline & $0.0523(14)$ & $0.0256(10)$ & $0.0259(9)$ & $0.0038(8)$ & $-0.0105(9)$ & $0.0215(10)$ \\
\hline $\mathrm{C} 2$ & $0.049(4)$ & $0.115(7)$ & $0.049(4)$ & $-0.049(5)$ & $0.023(3)$ & $-0.059(4)$ \\
\hline CD & $0.051(4)$ & $0.047(4)$ & $0.018(2)$ & $-0.008(2)$ & $-0.007(2)$ & $-0.031(3)$ \\
\hline
\end{tabular}


A
C22
$0.090(6) \quad 0.026(3)$
$0.092(6)$
$0.001(3)$
$-0.057(5) \quad-0.018(3)$ 
Hydrogen atom coordinates and isotropic atomic displacement parameters

Table S32. Hydrogen atomic coordinates and isotropic atomic displacement parameters $\left(\AA^{2}\right)$ for 12.

\begin{tabular}{|c|c|c|c|c|}
\hline & $\mathbf{x} / \mathbf{a}$ & $\mathbf{y} / \mathbf{b}$ & $\mathbf{z} / \mathbf{c}$ & $\mathbf{U}(\mathbf{e q})$ \\
\hline H6A & 0.9230 & 0.1145 & 0.6484 & 0.035 \\
\hline H6B & 0.7742 & 0.1645 & 0.7157 & 0.035 \\
\hline $\mathrm{H} 6 \mathrm{C}$ & 0.9434 & 0.1504 & 0.7484 & 0.035 \\
\hline $\mathrm{H} 7 \mathrm{~A}$ & 1.1063 & 0.2838 & 0.7782 & 0.045 \\
\hline $\mathrm{H} 7 \mathrm{~B}$ & 1.1105 & 0.4494 & 0.7840 & 0.045 \\
\hline $\mathrm{H} 7 \mathrm{C}$ & 1.2141 & 0.3603 & 0.7065 & 0.045 \\
\hline H8A & 0.9746 & 0.7582 & 0.6020 & 0.047 \\
\hline H8B & 1.1348 & 0.6450 & 0.5976 & 0.047 \\
\hline $\mathrm{H} 8 \mathrm{C}$ & 1.0488 & 0.6892 & 0.6928 & 0.047 \\
\hline H9A & 0.7978 & 0.6580 & 0.4609 & 0.035 \\
\hline H9B & 0.7496 & 0.7592 & 0.5429 & 0.035 \\
\hline H9C & 0.6357 & 0.6609 & 0.5182 & 0.035 \\
\hline H10A & 0.5507 & 0.4397 & 0.5685 & 0.027 \\
\hline H10B & 0.6235 & 0.2773 & 0.5990 & 0.027 \\
\hline $\mathrm{H} 10 \mathrm{C}$ & 0.6750 & 0.3447 & 0.5023 & 0.027 \\
\hline H11A & 0.5740 & 0.2832 & 0.9494 & 0.03 \\
\hline H11B & 0.6834 & 0.3974 & 0.9481 & 0.03 \\
\hline $\mathrm{H} 11 \mathrm{C}$ & 0.7432 & 0.2519 & 0.8986 & 0.03 \\
\hline H12A & 0.3432 & 0.5684 & 0.8063 & 0.025 \\
\hline H12B & 0.4377 & 0.6082 & 0.8830 & 0.025 \\
\hline $\mathrm{H} 12 \mathrm{C}$ & 0.3504 & 0.4764 & 0.9007 & 0.025 \\
\hline H15A & 0.5567 & 0.9835 & 0.5909 & 0.049 \\
\hline H15B & 0.6205 & 0.9754 & 0.6879 & 0.049 \\
\hline $\mathrm{H} 15 \mathrm{C}$ & 0.4844 & 1.1041 & 0.6592 & 0.049 \\
\hline H16A & 0.4522 & 0.9167 & 0.8259 & 0.064 \\
\hline H16B & 0.2811 & 0.8952 & 0.8144 & 0.064 \\
\hline H16C & 0.3239 & 1.0502 & 0.7939 & 0.064 \\
\hline H17A & 0.2304 & 1.0542 & 0.6354 & 0.048 \\
\hline H17B & 0.1903 & 0.9000 & 0.6642 & 0.048 \\
\hline $\mathrm{H} 17 \mathrm{C}$ & 0.3016 & 0.9217 & 0.5758 & 0.048 \\
\hline $\mathrm{H} 20 \mathrm{~A}$ & 1.0117 & 0.6330 & 1.0066 & 0.066 \\
\hline $\mathrm{H} 20 \mathrm{~B}$ & 1.0724 & 0.7771 & 0.9740 & 0.066 \\
\hline $\mathrm{H} 20 \mathrm{C}$ & 1.0721 & 0.6601 & 0.9037 & 0.066 \\
\hline $\mathrm{H} 21 \mathrm{~A}$ & 0.6620 & 0.8951 & 1.0012 & 0.061 \\
\hline $\mathrm{H} 21 \mathrm{~B}$ & 0.8181 & 0.9144 & 1.0395 & 0.061 \\
\hline $\mathrm{H} 21 \mathrm{C}$ & 0.7534 & 0.7706 & 1.0655 & 0.061 \\
\hline $\mathrm{H} 22 \mathrm{~A}$ & 0.9244 & 0.8593 & 0.8082 & 0.049 \\
\hline $\mathrm{H} 22 \mathrm{~B}$ & 0.9125 & 0.9716 & 0.8832 & 0.049 \\
\hline $\mathrm{H} 22 \mathrm{C}$ & 0.7588 & 0.9425 & 0.8466 & 0.049 \\
\hline
\end{tabular}




\begin{tabular}{lllll} 
H20D & 1.0783 & 0.6617 & 0.9010 & 0.096 \\
H20E & 1.0807 & 0.8186 & 0.9300 & 0.096 \\
H20F & 1.0294 & 0.7985 & 0.8331 & 0.096 \\
H21D & 0.7622 & 0.7401 & 1.0676 & 0.053 \\
H21E & 0.9059 & 0.8183 & 1.0649 & 0.053 \\
H21F & 0.9344 & 0.6506 & 1.0505 & 0.053 \\
H22D & 0.7770 & 0.9549 & 0.8592 & 0.098 \\
H22E & 0.8064 & 0.9868 & 0.9585 & 0.098 \\
H22F & 0.6590 & 0.9226 & 0.9423 & 0.098 \\
\hline
\end{tabular}

